\title{
Piracy in the Gulf of Guinea: Impacts to Maritime Transportation and Maritime Security
}

\author{
Nana Raymond Lawrence Ofosu-Boateng (Capt.) (Corresponding author) \\ College of Transport and Communications \\ Shanghai Maritime University \\ Tel: 86-131-2223-2396_E-mail: boatnana@gmail.com
}

Received: April 30, 2018 Accepted: May 7, 2018 Published: May 11, 2018

doi:10.5296/jad.v4i2.13124 URL: https://doi.org/10.5296/jad.v4i2.13124

\begin{abstract}
Piracy attacks in the Gulf of Guinea have taken a worrying trend. Unlike the Somali pirates, the pirates in the Gulf of Guinea target the cargoes, especially the oil laden tankers for their cargo. This disruption is impacting negatively on shipping in this sea lane. There are consequences to be suffered due to these pirate attacks on shipping in this region. This study delves into piracy and Maritime Transportation and Maritime Security in the Gulf of Guinea. The aim of this study is to analyse the impacts of piracy on different economic indicators and measurements with shipping as the vector of maritime transport. The economic indicators such as the liner shipping index, gross domestic product growth rate, imports growth rate, exports as a percentage of gross domestic product, and oil production as oil rents as a percent of gross domestic product. An Input-Output analysis to determine the interdependencies between variables was adopted. A Fixed Effects Model is used to show the relationship between pirate attacks and the countries distance from the Gulf of Guinea. A Correlation Analysis is conducted in respect of the countries and years based on the research question. A Chi Square test is conducted to test the hypotheses.
\end{abstract}

Keywords: Gulf of guinea, maritime security, maritime transportation, economic indicators 


\section{Introduction}

Piracy is commonly visualised through images of $18^{\text {th }}$ century privateers searching for ships projected to have large payouts. The $18^{\text {th }}$ century privateers were armed with guns, swords, knives, and cannons. Today's world still has privateers, albeit armed with more advanced weapons, such as automatic weapons and grenades, as well as more advanced ships. Because of the advanced tactics used by modern privateers, there have been headline cases of piracy, especially since the early 2000 s. However, piracy was deemed to be a limited problem prior to 2009 and considered to be a lower concern internationally. Common piracy attacks were attributed to small armed groups in territorial seas, such as in the Gulf of Guinea, in touted efforts to protect Somalia fishing resources (Bradford, 2005; Germond, 2011; Gilpin, 2007; Treves, 2009; Yang, Wang, Bonsall, \& Fang, 2009). Not only were these areas hunted by armed groups, but also foreign fisherman and individuals dumping waste products in coastal waters. However, there were also problems related to the inefficient and ineffective government, which increased opportunities for piracy. As a result, piracy became more prominent in different locations, as well as increasingly advanced, in part due to technological advancements and in part due to increased boldness by pirates (Treves, 2009).

\section{Research Aim}

Piracy attacks can be linked to international peace and security threats. The Security Council passed Resolution 733/1992, allowing Chapter VII to be invoked in order to address the piracy situation, citing security threats. The purpose of passing this resolution was to fight piracy within the framework of the United Nation's charter (Rosenberg \& Chung, 2008; Samuels, 2008; Treves, 2009). There are concerns regarding piracy law effectiveness, which are established through the United Nation Convention of the Law of the Sea, based on Articles 100 to 107 and 110. Due to the increasing rates of piracy in the Gulf of Guinea, there are increased concerns relating to the impact of these attacks (and attempted) attacks. The aim of this research study is to analyse the impact of piracy on different measurements and economic indicators, such as the liner shipping connectivity index, gross domestic product growth rate, imports growth rate, exports as a percent of gross domestic product, and oil production as oil rents as a percent of gross domestic product.

\section{Literature Review}

Maritime Piracy has been assigned diverse definitions. The most popular are the two given by United Nations Convention on the Law of the Sea (UNCLOS) Article 101 (1982) and the International Maritime Bureau (2008) which gave an encompassing definition. The UNCLOS definition limited piracy to be acts committed solely on the high seas but not most pirate attacks in the Gulf of Guinea happened in the territorial seas or anchorage. The IMB (2008) definition captures this omission by stating Piracy and Armed Robbery as follows:

"An act of boarding or attempting to board any vessel with the apparent intent to commit theft or any other crime and with the apparent intent or capability to use forces in the furtherance of the acts."

Collier (2007) stated that the Gulf of Guinea countries did not do enough to protect their 
coastline due to their inefficient and inadequate security systems. He further stated that, each attack by pirates was well planned and thought out before execution. That the pirates sometimes appeared heavily armed and had faster getaway speed boats and skiffs than the local coast guards or navies.

Tepp (2012) stated that the Gulf of Guinea had eight crucial tendencies which helped to fuel piracy in the Gulf of Guinea. These are: cultural acceptability, good political climate, insufficient security, weak judicial system, under resourced Law enforcement, reward promised, regional conflicts and good geography.

Luff \& Konin (2004) put the direct effects of maritime piracy in the Gulf of Guinea at some $\$ 250$ million and an estimated amount of $\$ 9$ billion saddled on the maritime industry through piracy. Doing trade along these dangerous waters had both direct and indirect effects on maritime transportation they stated.

Ramsbotham (2011) listed the following as some of the direct effects of piracy:

- Cargo fraud using so called phantom ships

- Penalties because of damaged or delayed goods

- Paying ransom money

- Investigative reports and their cost

- Entire ship loss

- Ship and Cargo damage due to an attack

- Ship cargo loss

Money has been a major catalyst in pushing the drive to engage in acts of piracy (Gjelsvik \& Bjorgo, 2012), Hansen (2012), Chalk (2009). Clark (2009) stated that 'opportunity' was a huge factor for an act of piracy to be committed.

\section{Research Questions and Hypotheses}

Piracy attacks in the Gulf of Guinea have become increasingly pervasive in recent years. According to the International Maritime Bureau (2015), Nigeria has been the most affected country due to piracy attacks and the most affected area is the Gulf of Guinea. As a result, piracy has a negative impact on maritime transportation and maritime security, particularly in relation to the development of oil production in the area Bowden (2010). The research questions and hypotheses to be addressed include:

Q1. To what extent does piracy impact the Gulf of Guinea in relation to the liner shipping connectivity index?

$\mathrm{H}_{0}$ : Piracy has a significant impact on the Gulf of Guinea in relation to the liner shipping connectivity index.

$\mathrm{H}_{1}$ : Piracy has no impact on the Gulf of Guinea in relation to the liner shipping connectivity 


\section{Macrothink}

index.

Q2. To what extent does piracy impact the Gulf of Guinea in relation to gross domestic product growth rate?

$\mathrm{H}_{0}$ : Piracy has a significant impact on the Gulf of Guinea in relation to gross domestic product growth rate.

$\mathrm{H}_{2}$ : Piracy has no impact on the Gulf of Guinea in relation to gross domestic product growth rate.

Q3. To what extent does piracy impact the Gulf of Guinea in relation to imports growth rate?

$\mathrm{H}_{0}$ : Piracy has a significant impact on the Gulf of Guinea in relation to imports growth rate.

$\mathrm{H}_{3}$ : Piracy has no impact on the Gulf of Guinea in relation to imports growth rate.

Q4. To what extent does piracy impact the Gulf of Guinea in relation to exports as a percent of gross domestic product?

$\mathrm{H}_{0}$ : Piracy has a significant impact on the Gulf of Guinea in relation to exports as a percent of gross domestic product.

$\mathrm{H}_{4}$ : Piracy has no impact on the Gulf of Guinea in relation to exports as a percent of gross domestic product.

Q5. To what extent does piracy impact the Gulf of Guinea in relation to oil production as oil rents as a percent of gross domestic product?

$\mathrm{H}_{0}$ : Piracy has a significant impact on the Gulf of Guinea in relation to oil production as oil rents as a percent of gross domestic product.

$\mathrm{H}_{5}$ : Piracy has no impact on the Gulf of Guinea in relation to oil production as oil rents as a Percent of gross domestic product.

\section{Data Collection}

The panel data used for this study was obtained from the International Maritime Bureau and the World Bank. Panel data was used because of the consideration of all data uses. The time period assessed is 2007 to 2016. Raw data is shown in the appendix. Data includes coastal countries of Africa. Microsoft Excel was used for data analysis purposes.

\subsection{Data Variables}

\subsubsection{Research Question One}

The first research question asked: To what extent does piracy impact the Gulf of Guinea in relation to the liner shipping connectivity index? The variables being used in relation to this research question are the average of attacks and liner shipping connectivity per year and per country. 


\subsubsection{Research Question Two}

The second research question asked: To what extent does piracy impact the Gulf of Guinea in relation to gross domestic product growth rate? The variables being used in relation to this research question are the average of attacks and growth domestic product growth rate per year and per country.

\subsubsection{Research Question Three}

The third research question asked: To what extent does piracy impact the Gulf of Guinea in relation to imports growth rate? The variables being used in relation to this research question are the average of attacks and imports growth rate per year and per country.

\subsubsection{Research Question Four}

The fourth research question asked: To what extent does piracy impact the Gulf of Guinea in relation to exports as a percent of gross domestic product? The variables being used in relation to this research question are the average of attacks and exports as a percent of gross domestic product per year and per country.

\section{Variables Justification}

According to Onuoha (2009), African waterways are some of the most dangerous waters for vessels to cross. These attacks have led researchers to believe that piracy is the largest industry in Somalia, which has led to further attacks. In fact, based on Maritime Piracy Data, there were 3,413 piracy and/or armed robbery events against ships between 2000 and 2009 (Coggins, 2012). This number has increased, despite how maritime concerns have become high on national and international security agendas. In fact, as of 2009 , there was a steep increase in maritime threats, leading to increased awareness of threats to both humanitarian and commercial interests, as well as important resources (Vreÿ, 2009). In fact, it is recognised that "maritime piracy imposes direct costs on the immediate victims of the attacks - the crews, the ships and their cargoes, and the shipping companies. Merchant seamen may be injured or killed; ships and cargoes stolen, and higher insurance rates and operating costs borne by companies. At the same time, the indirect costs of maritime piracy are substantial, particularly in humanitarian terms" (Nincic, 2009). Moreover, the threat of piracy has increased, such as in relation to the Tanzanian sea route, which has led to jeopardise to commercial shipping. In Somalia, piracy has hindered humanitarian efforts, such as the delivery of aid for a substantial part of the population. In Nigeria, piracy has severely impacted the fishing industry and regional trade.

Piracy in Somalia and Nigeria alone hinders living situations for many people Nincic (2009). According to Sullivan (2010), "maritime piracy impacts international business and disrupts the global supply chain."Neethling (2010) argued that there is a close interface between both globalisation and maritime trade, further arguing that "most security challenges confronting Africa have their origin in the lack or failure of governance as states are the primary actors and agents of good order at sea." This is concerning as "container traffic in West African ports has grown 14 percent annually since 1995" (Osinowo, 2015). Moreover, the Gulf of 


\section{Macrothink}

Guinea represents the main transit hub and facilitator to the region, as well as for global energy supplies.

According to Osinowo (2015), in 2012, the Gulf of Guinea surpassed that of the Gulf of Aden (infamous for high-seas hijackings) as the region with the highest number of reported piracy attacks in the world. These attacks also tended to be more violent. Given the limited maritime security presence off the West African coast, South American narcotics traffickers have found the region an attractive transit route to Europe. Oil theft and illegal bunkering plague the Gulf of Guinea. Nigeria alone loses between 40,000 and 100,000 barrels a day due to theft. With 40 percent of the region $\mathrm{s}$ annual catch estimated to be illegal, unregulated, or unreported, West Africa's waters also endure the highest level of illegal fishing in the world. Trade partners have taken note. In 2013, almost all of the estimated $\$ 10.2$ billion worth of regional trade with the United Kingdom moving through the Gulf of Guinea was declared at risk of theft."

\section{Descriptive Statistics}

The goal of descriptive statistics is to describe the data being presented. In this case, data is being described in terms of different variables - piracy attacks, liner shipping connectivity index, growth domestic product growth rate, imports growth rate, exports as a percent of gross domestic product, and oil production as oil rents as a percent of gross domestic product. For this analysis, the descriptive statistics being considered are mean, median, minimum, maximum, and standard deviation (Mann, 1995; Trochim, 2006).

\subsection{Input-Output Analysis}

The input-output model is a quantitative economic analysis, representing the interdependencies between different variables (Clark, 1984). This analysis type was developed by Wassily Leontief (1906-1999) and was built alongside work conducted by Francois Quesnay, Léon Walras, Alexander Bogdanov, L. N. Kritsman, T. F. Remington, Vladimir Groman, and Vladimir Bazarov. Leontief is noted for being influenced by classical economics - such as works by Karl Marx and Jean Charles Léonard de Sismondi - and was the first to use a matrix representation of the economy (Belykh, 1989). The focus of the model is the inter-relationships within the economy, showing both independence and dependence among sectors (Loucks \& Whitney, 1973; Nikaido, 1970). The following table shows the input requirements:

Table 1. Input requirements

\begin{tabular}{lll}
\hline Variable & Piracy Attacks & Variable Y(Note 1) \\
\hline Piracy Attacks & 0 & MAXIMUM (Note 2) \\
Variable Y & 1 - MAXIMUM & 0 \\
\hline
\end{tabular}




\section{Macrothink

It is recognised that for this study, the input requirements for Variable Y will vary based on the analysis being conducted (based on the research question and related variables) and will be considered a percent of gross domestic product. The gross domestic product average was calculated based on information from the appendix and is shown in the following table:

Table 2. Average Gross Domestic Product

\begin{tabular}{llll}
\hline Country & Average gross domestic product & Year & Average gross domestic product \\
\hline Angola & 85.92 & 2007 & 36.53 \\
Benin & 7.46 & 2008 & 39.00 \\
Cameroon & 25.49 & 2009 & 30.91 \\
Dem. Congo Rep. & 17.56 & 2010 & 43.20 \\
Egypt & 219.83 & 2011 & 45.12 \\
Equatorial Guinea & 1.17 & 2012 & 46.96 \\
Eritrea & 16.63 & 2013 & 46.26 \\
Ghana & 0.88 & 2014 & 51.70 \\
Guinea & 4.92 & 2015 & 53.56 \\
Guinea Bissau & 36.43 & 2016 & 54.33 \\
Ivory Coast & 27.25 & & \\
Kenya & 42.97 & & \\
Liberia & 1.38 & & \\
Madagascar & 9.11 & & \\
Mauritania & 4.70 & & \\
Morocco & 97.26 & & \\
Mozambique & 11.13 & & \\
Nigeria & 382.21 & & \\
Seychelles & 1.07 & & \\
Sierra Leone & 2.91 & & \\
Somalia & 0.00 & & \\
Tanzania & 34.46 & & \\
Togo & 3.40 & & \\
\hline & & & \\
& & & \\
& & & \\
& & & \\
\hline
\end{tabular}

The gross domestic product results are compared with the average for the entire dataset in the following figure: 


\section{IIMacrothink}

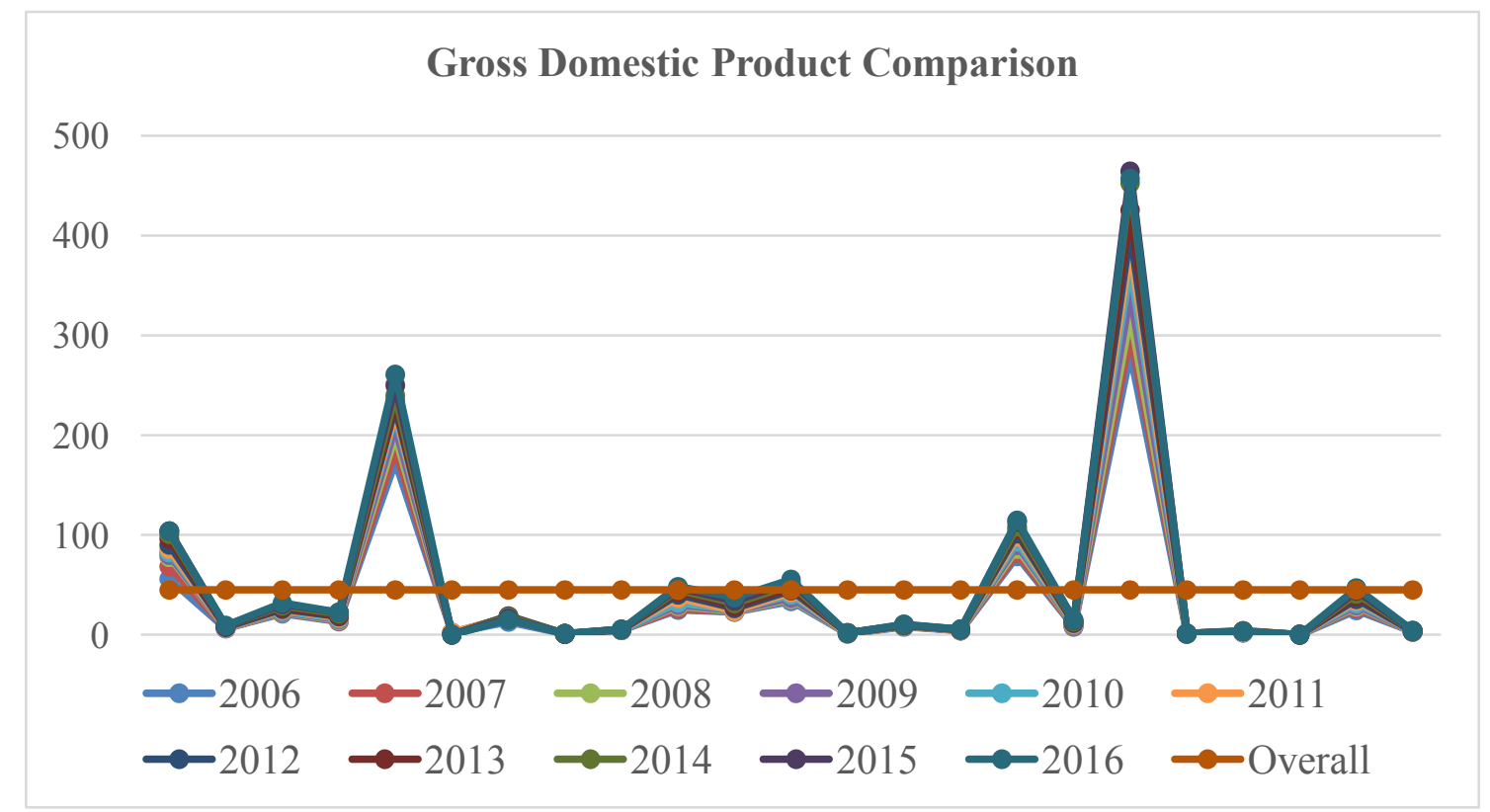

Figure 1. Gross Domestic Product Comparison Source: Author's Compilation. (2018)

Within the requirements table, the amount required will be the percent times the gross domestic product applicable from the prior table. The formula for the indirect requirements for the first variable (piracy attacks) is

$$
\begin{aligned}
& a_{1}-X a_{b}=1 \\
& a_{b}-X a_{1}=0
\end{aligned}
$$

Where $a_{1}=$ output of piracy attacks; $a_{b}=$ output of Variable $Y$; and $X=$ requirement (based on the variable)

\subsection{Fixed Effects Model}

The fixed effects model is used to show the relationship of piracy attacks and the country's distance from the Gulf of Guinea. The variables used are distance and total distance (see appendix) to determine the relationship held in terms of piracy. For this study; however, the formula will be altered slightly to include piracy attacks, as shown

$$
a=\frac{x / y}{z}
$$

where $x$ = country distance; $y=$ total distance; $z=$ piracy attacks (Baetschmann, Staub, \& Winkelmann, 2015; Blanchard \& Landier, 2002; Chen, Schmidt, \& Wang, 2014; Hausman \& de Leeuw, 1978; Subramanian, Jones, Kaddour, \& Krieger, 2009). 


\section{Macrothink}

\subsection{Correlation Analysis}

The correlation is designed to allow the variable relationship to be shown. The results allow for a single number ("Computing the Pearson Correlation Coefficient," 2003, "Data Analysis - Pearson's Correlation Coefficient," 2015; Sedgwick, 2012). Positive relationships have an upwards slope, whereas negative relationships have a downwards slope. Generally, a positive relationship requires higher scores being paired. The formula for the correlation is:

$$
r=\frac{N \sum x y-\left(\sum x\right)\left(\sum y\right)}{\sqrt{\left[N \sum x^{2}-\left(\sum x\right)^{2}\right]\left[N \sum y^{2}-\left(\sum y\right)^{2}\right]}}
$$

Where $N=$ sample size; $\sum x y=$ sum of products; $\sum x=$ sum of $x$ values; $\sum y=$ sum of $y$ values; $\sum x^{2}=$ sum of $x$-squared values; and $\sum y^{2}=$ sum of $y$-squared values. The correlation will be between -1.0 and 1.0 (Sedgwick, 2012).

\subsection{Chi Square Test}

The final test is the chi square test, which is designed for testing the hypotheses. If the test statistic is under the critical value, the null hypothesis is accepted. If the test statistic is over the critical value, the null hypothesis is rejected. The chi square test uses both observations and expected values. The formula for expected values is

$$
\frac{(x \times y)}{z}
$$

Where $x=$ the row total; $y=$ the column total; and $z=$ the sum of all values.

The formula for the test statistics is

$$
\sum \frac{(x-y)^{2}}{y}
$$

where $x=$ observed value and $y=$ the expected value ("Chi Square Test," n.d., Critical Values of the Chi-Square Distribution., n.d.; McHugh, 2013; Niedermeier, Wang, \& Zhang, 2016; Penn State Science, 2015; Test, 2015; University of Regina, n.d.). The chi square test is being used for significance in order to provide definitive answers to the hypotheses.

\section{Application to the Data}

\subsection{Mean}

The mean is the average of the data. It is calculated as:

$$
\frac{\sum x}{n}
$$

where $\sum x=$ sum of all values and $n=$ number of values (Babbie, 2010). The mean is found 
for both country and year. The data was inputted into Excel (Note 3 ) and the average output is shown below:

Table 3. Averages by country

\begin{tabular}{|c|c|c|c|c|c|c|}
\hline & $\begin{array}{l}\text { Piracy } \\
\text { attacks }\end{array}$ & $\begin{array}{l}\text { Liner shipping } \\
\text { connectivity } \\
\text { index }\end{array}$ & $\begin{array}{l}\text { Gross domestic } \\
\text { product growth } \\
\text { rate }\end{array}$ & Imports & Exports & $\begin{array}{l}\text { Oil } \\
\text { production }\end{array}$ \\
\hline Angola & 0.70 & 14.97 & 6.59 & 0.00 & 56.59 & 33.24 \\
\hline Benin & 2.40 & 14.34 & 4.27 & 9.30 & 25.46 & 0.00 \\
\hline Cameroon & 1.30 & 12.00 & 4.19 & 9.03 & 20.34 & 5.06 \\
\hline $\begin{array}{l}\text { Dem.Congo } \\
\text { Rep. }\end{array}$ & 4.50 & 9.49 & 5.10 & 3.66 & 55.51 & 20.06 \\
\hline Egypt & 2.20 & 54.92 & 4.19 & 5.03 & 20.14 & 6.93 \\
\hline $\begin{array}{l}\text { Equatorial } \\
\text { Guinea }\end{array}$ & 0.10 & 5.41 & 1.67 & 2.95 & 73.44 & 28.70 \\
\hline Eritrea & 0.10 & 2.98 & 0.64 & 0.26 & 3.39 & 0.00 \\
\hline Ghana & 2.50 & 18.92 & 6.84 & 11.65 & 34.39 & 2.36 \\
\hline Guinea & 2.80 & 7.49 & 2.42 & 7.58 & 28.56 & 0.00 \\
\hline Guinea Bissau & 0.10 & 4.19 & 3.54 & 6.82 & 21.53 & 0.00 \\
\hline Ivory Coast & 2.40 & 19.54 & 5.15 & 5.17 & 44.96 & 2.44 \\
\hline Kenya & 1.40 & 11.98 & 5.23 & 5.85 & 19.86 & 0.00 \\
\hline Liberia & 0.60 & 6.26 & 5.21 & 2.40 & 30.24 & 0.00 \\
\hline Madagascar & 0.10 & 9.66 & 2.69 & 5.91 & 28.80 & 0.00 \\
\hline Mauritania & 0.10 & 7.02 & 3.32 & 6.45 & 45.48 & 3.42 \\
\hline Morocco & 0.50 & 48.96 & 3.85 & 5.10 & 33.63 & 0.01 \\
\hline Mozambique & 1.20 & 9.30 & 6.67 & 17.59 & 31.82 & 0.10 \\
\hline Nigeria & 26.60 & 21.07 & 4.99 & 4.92 & 23.95 & 13.37 \\
\hline Seychelles & 0.10 & 6.49 & 4.50 & 0.00 & 75.09 & 0.00 \\
\hline Sierra Leone & 0.70 & 6.07 & 5.43 & 19.47 & 21.04 & 0.00 \\
\hline Somalia & 48.90 & 4.35 & 0.00 & 0.00 & 5.84 & 0.00 \\
\hline Tanzania & 3.50 & 6.40 & 6.70 & 9.99 & 19.20 & 0.00 \\
\hline Togo & 3.40 & 3.57 & 4.19 & 18.82 & 41.86 & 0.00 \\
\hline
\end{tabular}

It is noted that the lowest incidences of piracy attacks are 0.10, occurring in Equatorial Guinea, Eritrea, Madagascar, Mauritania, and Seychelles. The highest rate of piracy attacks is 
48.90, occurring in Nigeria. The lowest Liner Shipping connectivity index is 2.98 in Eritrea, whereas the highest Liner Shipping connectivity index is 54.92 in Egypt. The lowest gross domestic product growth rate is 0.00 in Somalia, whereas the highest gross domestic product growth rate is 6.70 in Tanzania. The lowest imports are 0.00 in Angola and Somalia, whereas the highest imports are 19.47 in Sierra Leone. The lowest exports are 3.39 in Eritrea, whereas the highest exports are 73.44 in Equatorial Guinea. The lowest oil production is 0.00 in Benin, Entree, Guinea, Guinea Bissau, Kenya, Liberia, Madagascar, Seychelles, Sierra Leone, Somalia, Tanzania, and Togo. However, the highest oil production is in Angola.

Table 4. Averages by year

\begin{tabular}{lcccccccccc}
\hline & $\mathbf{2 0 0 7}$ & $\mathbf{2 0 0 8}$ & $\mathbf{2 0 0 9}$ & $\mathbf{2 0 1 0}$ & $\mathbf{2 0 1 1}$ & $\mathbf{2 0 1 2}$ & $\mathbf{2 0 1 3}$ & $\mathbf{2 0 1 4}$ & $\mathbf{2 0 1 5}$ & $\mathbf{2 0 1 6}$ \\
\hline $\begin{array}{l}\text { Piracy attacks } \\
\begin{array}{l}\text { Liner shipping connectivity } \\
\text { index }\end{array}\end{array}$ & 4.65 & 4.26 & 5.83 & 7.87 & 9.43 & 5.35 & 3.00 & 1.96 & 1.22 & 2.61 \\
$\begin{array}{l}\text { Gross Domestic } \\
\text { Growth Rate }\end{array}$ & 9.44 & 11.25 & 12.15 & 11.79 & 12.60 & 13.80 & 13.58 & 15.22 & 16.63 & 16.31 \\
$\begin{array}{l}\text { Imports } \\
\text { Exports }\end{array}$ & 6.15 & 4.71 & 2.88 & 3.96 & 5.18 & 5.23 & 5.26 & 4.14 & 2.09 & 2.73 \\
Oil production & 14.94 & 7.22 & 1.86 & 11.24 & 10.27 & 10.05 & 7.99 & 2.68 & 1.09 & 1.32 \\
\hline & 37.36 & 36.44 & 31.84 & 34.00 & 35.97 & 35.45 & 33.98 & 32.93 & 27.14 & 25.81 \\
& 7.83 & 8.20 & 4.29 & 5.41 & 6.68 & 6.31 & 5.28 & 4.39 & 1.90 & 0.00 \\
\hline
\end{tabular}

The lowest piracy attacks were 1.22 in 2015, whereas the highest piracy attacks were 9.43 in 2011. The lowest Liner Shipping connectivity index were 9.44 in 2007, whereas the highest Liner Shipping connectivity index were 16.63 in 2015. The lowest gross domestic product growth rate was 2.09 in 2015, whereas the highest gross domestic product growth rate was 6.15 in 2007. The lowest imports were 1.09 in 2015, whereas the highest imports were 14.94 in 2007. The lowest exports were 25.81 in 2016, whereas the highest export rates were 37.36 in 2007. The lowest oil production was 0.00 in 2016, whereas the highest oil production was 8.20 in 2008.

\subsection{Median, Minimum, and Maximum}

The median, minimum, and maximum is only being shown in relation to the countries. The median is the number that occurs in the middle of a dataset that has been ordered numerically. The minimum is the smallest data point. The maximum is the largest data point (Nick, 2007). To do this, the data was inputted into Excel (Note 4) and the following output was observed based on the variable: 
Table 5. Median, minimum, and maximum

\begin{tabular}{llllllll}
\hline & $\begin{array}{l}\text { Piracy } \\
\text { attacks }\end{array}$ & $\begin{array}{l}\text { Liner shipping } \\
\text { connectivity } \\
\text { index }\end{array}$ & $\begin{array}{l}\text { Gross } \\
\text { Product } \\
\text { Rate }\end{array}$ & $\begin{array}{l}\text { Domestic } \\
\text { Growth }\end{array}$ & Imports & Exports & $\begin{array}{l}\text { Oil } \\
\text { production }\end{array}$ \\
\hline Median & 1 & 8.42 & 4.56 & 3.58 & 28.89 & 0.00 \\
Minimum & 0 & 0.00 & -20.49 & -32.89 & 0.00 & 0.00 \\
Maximum & 160 & 68.28 & 20.72 & 96.67 & 107.99 & 48.50 \\
\hline
\end{tabular}

\subsection{Standard Deviation}

Standard deviation is important because it shows the distance from the mean a data point is.

This is calculated using:

$s=\sqrt{\frac{1}{N-1} \sum_{i=1}^{N}\left(x_{i}-\bar{x}\right)^{2}}$

where $N=$ sample size; $x_{i}=$ data point; and $\bar{x}=$ mean (Hebl, 2015; Mann, 1995; Nick, 2007; Trochim, 2006). The mean is found for both country and year. The data was inputted into Excel (Note 5) and the average output is shown below:

Table 6 . Standard deviation by country

\begin{tabular}{lllllll}
\hline & $\begin{array}{l}\text { Piracy } \\
\text { attacks }\end{array}$ & $\begin{array}{l}\text { Liner shipping } \\
\text { connectivity } \\
\text { index }\end{array}$ & $\begin{array}{l}\text { Gross Domestic } \\
\text { Product } \\
\text { Growth Rate }\end{array}$ & Imports & Exports & $\begin{array}{l}\text { Oil } \\
\text { production }\end{array}$ \\
\hline Angola & 0.82 & 3.03 & 6.70 & 0.00 & 14.83 & 17.62 \\
Benin & 6.22 & 6.25 & 1.87 & 13.26 & 4.02 & 0.00 \\
Cameroon & 1.64 & 2.64 & 1.34 & 7.13 & 2.79 & 2.76 \\
Dem. Congo & 2.59 & 1.34 & 2.38 & 14.12 & 7.50 & 9.35 \\
Rep. & & & 1.94 & 13.70 & 7.43 & 3.63 \\
Egypt & 2.74 & 3.15 & 10.06 & 23.04 & 11.71 & 13.97 \\
Equatorial & 0.32 & 6.09 & 4.58 & 13.54 & 4.57 & 0.00 \\
Guinea & & & 3.37 & 17.53 & 6.94 & 2.66 \\
Eritrea & 0.32 & 2.41 & 2.00 & 14.08 & 2.84 & 0.00 \\
Ghana & 1.96 & 1.60 & & & & \\
Guinea & 2.10 & 2.12 & & & & \\
\hline
\end{tabular}




\begin{tabular}{|c|c|c|c|c|c|c|}
\hline Guinea Bissau & 0.32 & 1.23 & 2.93 & 21.89 & 4.86 & 0.00 \\
\hline Ivory Coast & 1.65 & 0.62 & 4.83 & 15.71 & 6.77 & 1.61 \\
\hline Kenya & 1.17 & 4.73 & 2.20 & 5.86 & 2.63 & 0.00 \\
\hline Liberia & 0.70 & 0.94 & 4.03 & 6.77 & 17.19 & 0.00 \\
\hline Madagascar & 0.32 & 1.44 & 3.12 & 12.88 & 3.69 & 0.00 \\
\hline Mauritania & 0.32 & 1.90 & 2.42 & 13.63 & 6.83 & 2.64 \\
\hline Morocco & 0.53 & 1.14 & 1.39 & 7.50 & 2.23 & 0.01 \\
\hline Mozambique & 1.03 & 18.41 & 1.05 & 18.82 & 1.73 & 0.05 \\
\hline Nigeria & 11.04 & 1.09 & 2.74 & 32.56 & 12.13 & 8.39 \\
\hline Seychelles & 0.32 & 4.86 & 3.85 & 0.00 & 40.21 & 0.00 \\
\hline Sierra Leone & 0.82 & 1.46 & 10.59 & 31.22 & 7.39 & 0.00 \\
\hline Somalia & 58.87 & 1.23 & 0.00 & 0.00 & 7.54 & 0.00 \\
\hline Tanzania & 5.02 & 1.17 & 1.09 & 16.08 & 1.55 & 0.00 \\
\hline Togo & 4.77 & 0.71 & 1.23 & 32.55 & 5.16 & 0.00 \\
\hline
\end{tabular}

Table7. Standard deviation by year

\begin{tabular}{|c|c|c|c|c|c|c|c|c|c|c|}
\hline & 2007 & 2008 & 2009 & 2010 & 2011 & 2012 & 2013 & 2014 & 2015 & 2016 \\
\hline Piracy attacks & 10.83 & 9.26 & 18.07 & 30.20 & 34.69 & 11.68 & 6.78 & 4.15 & 3.04 & 7.83 \\
\hline $\begin{array}{l}\text { Liner } \\
\text { Shipping } \\
\text { Connectivity } \\
\text { Index }\end{array}$ & 407.84 & 407.73 & 407.77 & 408.07 & 408.14 & 408.12 & 408.38 & 408.31 & 408.26 & 408.50 \\
\hline $\begin{array}{l}\text { Gross } \\
\text { Domestic } \\
\text { Product } \\
\text { Growth Rate }\end{array}$ & 5.05 & 5.36 & 2.70 & 3.71 & 3.64 & 3.67 & 4.66 & 2.76 & 6.10 & 3.88 \\
\hline Imports & 23.02 & 11.23 & 15.57 & 11.05 & 23.04 & 22.97 & 19.59 & 10.60 & 14.04 & 9.39 \\
\hline Exports & 24.67 & 24.82 & 23.71 & 23.81 & 22.77 & 22.07 & 19.58 & 18.23 & 14.97 & 14.55 \\
\hline $\begin{array}{l}\text { Oil } \\
\text { production }\end{array}$ & 15.13 & 15.78 & 8.20 & 10.83 & 12.47 & 11.88 & 10.03 & 8.35 & 4.01 & 0.00 \\
\hline
\end{tabular}

\subsection{Input-Output Analysis}

The input-output analysis is conducted based on a direct and indirect requirements table. The input requirements, based on the research questions are: 
Table 8. Requirements table

\begin{tabular}{|c|c|c|c|}
\hline \multirow{3}{*}{$\begin{array}{l}\text { Question } \\
1\end{array}$} & & Piracy attacks & Liner Shipping Connectivity Index \\
\hline & Piracy attacks & 0 & 68.28 \\
\hline & $\begin{array}{l}\text { Liner Shipping Connectivity } \\
\text { Index }\end{array}$ & 31.62 & 0 \\
\hline \multirow{3}{*}{$\begin{array}{l}\text { Question } \\
2\end{array}$} & & Piracy attacks & $\begin{array}{l}\text { Gross Domestic Product } \text { Growth } \\
\text { Rate }\end{array}$ \\
\hline & Piracy attacks & 0 & 20.72 \\
\hline & $\begin{array}{l}\text { Gross Domestic Product Growth } \\
\text { Rate }\end{array}$ & 79.28 & 0 \\
\hline \multirow{3}{*}{$\begin{array}{l}\text { Question } \\
3\end{array}$} & & Piracy attacks & Imports Growth Rate \\
\hline & Piracy attacks & 0 & 96.67 \\
\hline & Imports Growth Rate & 3.333 & 0 \\
\hline \multirow{3}{*}{$\begin{array}{l}\text { Question } \\
4\end{array}$} & & Piracy attacks & Exports \\
\hline & Piracy attacks & 0 & 107.99 \\
\hline & Exports & -7.99 & 0 \\
\hline \multirow{3}{*}{$\begin{array}{l}\text { Question } \\
5\end{array}$} & & Piracy attacks & Oil production \\
\hline & Piracy attacks & 0 & 48.50 \\
\hline & Oil production & 51.50 & 0 \\
\hline
\end{tabular}

The following table shows the gross domestic product results, based on the previous table and relevant average gross domestic product (which must meet the average gross domestic product of the entire system):

Table 9. Gross domestic product results (based on country)

\begin{tabular}{llllll}
\hline & Question1 & Question 2 & Question 3 & Question 4 & Question 5 \\
\hline Angola & 0.00 & 58.67 & 0.00 & 17.80 & 0.00 \\
& 27.17 & 0.00 & 68.12 & 0.00 & 2.86 \\
\hline Benin & 0.00 & 5.09 & 0.00 & 1.55 & 0.00 \\
& 2.36 & 0.00 & 5.91 & 0.00 & 0.25 \\
\hline Cameroon & 0.00 & 17.40 & 0.00 & 5.28 & 0.00 \\
& 8.06 & 0.00 & 20.21 & 0.00 & 0.85 \\
\hline Dem. Congo Rep. & 0.00 & 11.99 & 0.00 & 3.64 & 0.00 \\
& 5.55 & 0.00 & 13.92 & 0.00 & 0.59 \\
\hline
\end{tabular}




\begin{tabular}{|c|c|c|c|c|c|}
\hline \multirow[t]{2}{*}{ Egypt } & 0.00 & 150.10 & 0.00 & 45.55 & 0.00 \\
\hline & 69.51 & 0.00 & 174.28 & 0.00 & 7.33 \\
\hline \multirow[t]{2}{*}{ Equatorial Guinea } & 0.00 & 0.80 & 0.00 & 0.24 & 0.00 \\
\hline & 0.37 & 0.00 & 0.93 & 0.00 & 0.04 \\
\hline \multirow{2}{*}{ Eritrea } & 0.00 & 11.35 & 0.00 & 3.45 & 0.00 \\
\hline & 5.26 & 0.00 & 13.18 & 0.00 & 0.55 \\
\hline \multirow[t]{2}{*}{ Ghana } & 0.00 & 0.60 & 0.00 & 0.18 & 0.00 \\
\hline & 0.28 & 0.00 & 0.70 & 0.00 & 0.03 \\
\hline \multirow[t]{2}{*}{ Guinea } & 0.00 & 3.36 & 0.00 & 1.02 & 0.00 \\
\hline & 1.56 & 0.00 & 3.90 & 0.00 & 0.16 \\
\hline \multirow[t]{2}{*}{ Guinea Bissau } & 0.00 & 24.87 & 0.00 & 7.55 & 0.00 \\
\hline & 11.52 & 0.00 & 28.88 & 0.00 & 1.21 \\
\hline \multirow{2}{*}{ Ivory Coast } & 0.00 & 18.61 & 0.00 & 5.65 & 0.00 \\
\hline & 8.62 & 0.00 & 21.60 & 0.00 & 0.91 \\
\hline \multirow[t]{2}{*}{ Kenya } & 0.00 & 29.34 & 0.00 & 8.90 & 0.00 \\
\hline & 13.59 & 0.00 & 34.07 & 0.00 & 1.43 \\
\hline \multirow[t]{2}{*}{ Liberia } & 0.00 & 0.94 & 0.00 & 0.29 & 0.00 \\
\hline & 0.44 & 0.00 & 1.09 & 0.00 & 0.05 \\
\hline \multirow[t]{2}{*}{ Madagascar } & 0.00 & 6.22 & 0.00 & 1.89 & 0.00 \\
\hline & 2.88 & 0.00 & 7.22 & 0.00 & 0.30 \\
\hline \multirow[t]{2}{*}{ Mauritania } & 0.00 & 3.21 & 0.00 & 0.97 & 0.00 \\
\hline & 1.49 & 0.00 & 3.73 & 0.00 & 0.16 \\
\hline \multirow[t]{2}{*}{ Morocco } & 0.00 & 66.41 & 0.00 & 20.15 & 0.00 \\
\hline & 30.75 & 0.00 & 77.11 & 0.00 & 3.24 \\
\hline \multirow[t]{2}{*}{ Mozambique } & 0.00 & 7.60 & 0.00 & 2.31 & 0.00 \\
\hline & 3.52 & 0.00 & 8.82 & 0.00 & 0.37 \\
\hline \multirow[t]{2}{*}{ Nigeria } & 0.00 & 260.97 & 0.00 & 79.19 & 0.00 \\
\hline & 120.85 & 0.00 & 303.02 & 0.00 & 12.74 \\
\hline \multirow[t]{2}{*}{ Seychelles } & 0.00 & 0.73 & 0.00 & 0.22 & 0.00 \\
\hline & 0.34 & 0.00 & 0.85 & 0.00 & 0.04 \\
\hline \multirow[t]{2}{*}{ Sierra Leone } & 0.00 & 1.99 & 0.00 & 0.60 & 0.00 \\
\hline & 0.92 & 0.00 & 2.31 & 0.00 & 0.10 \\
\hline \multirow[t]{2}{*}{ Somalia } & 0.00 & 0.00 & 0.00 & 0.00 & 0.00 \\
\hline & 0.00 & 0.00 & 0.00 & 0.00 & 0.00 \\
\hline
\end{tabular}




\begin{tabular}{llllll}
\hline Tanzania & 0.00 & 23.53 & 0.00 & 7.14 & 0.00 \\
& 10.90 & 0.00 & 27.32 & 0.00 & 1.15 \\
\hline Togo & 0.00 & 2.32 & 0.00 & 0.70 & 0.00 \\
& 1.08 & 0.00 & 2.70 & 0.00 & 0.11 \\
\hline
\end{tabular}

Table 10. Gross domestic product results (based on year)

\begin{tabular}{|c|c|c|c|c|c|c|c|c|c|c|}
\hline \multirow{2}{*}{2007} & \multicolumn{2}{|c|}{ Question 1} & \multicolumn{2}{|c|}{ Question 2} & \multicolumn{2}{|c|}{ Question 3} & \multicolumn{2}{|c|}{ Question 4} & \multicolumn{2}{|c|}{ Question 5} \\
\hline & 0.00 & 24.94 & 0.00 & 7.57 & 0.00 & 35.31 & 0.00 & 39.45 & 0.00 & 17.72 \\
\hline & 11.55 & 0.00 & 28.96 & 0.00 & 1.22 & 0.00 & -2.92 & 0.00 & 18.81 & 0.00 \\
\hline \multirow[t]{2}{*}{2008} & 0.00 & 26.63 & 0.00 & 8.08 & 0.00 & 37.70 & 0.00 & 42.12 & 0.00 & 18.92 \\
\hline & 12.33 & 0.00 & 30.92 & 0.00 & 1.30 & 0.00 & -3.12 & 0.00 & 20.09 & 0.00 \\
\hline \multirow[t]{2}{*}{2009} & 0.00 & 21.11 & 0.00 & 6.40 & 0.00 & 29.88 & 0.00 & 33.38 & 0.00 & 14.99 \\
\hline & 9.77 & 0.00 & 24.51 & 0.00 & 1.03 & 0.00 & -2.47 & 0.00 & 15.92 & 0.00 \\
\hline \multirow[t]{2}{*}{2010} & 0.00 & 29.50 & 0.00 & 8.95 & 0.00 & 41.76 & 0.00 & 46.65 & 0.00 & 20.95 \\
\hline & 13.66 & 0.00 & 34.25 & 0.00 & 1.44 & 0.00 & -3.45 & 0.00 & 22.25 & 0.00 \\
\hline \multirow[t]{2}{*}{2011} & 0.00 & 30.81 & 0.00 & 9.35 & 0.00 & 43.62 & 0.00 & 48.73 & 0.00 & 21.88 \\
\hline & 14.27 & 0.00 & 35.77 & 0.00 & 1.50 & 0.00 & -3.61 & 0.00 & 23.24 & 0.00 \\
\hline \multirow[t]{2}{*}{2012} & 0.00 & 32.06 & 0.00 & 9.73 & 0.00 & 45.40 & 0.00 & 50.71 & 0.00 & 22.78 \\
\hline & 14.85 & 0.00 & 37.23 & 0.00 & 1.57 & 0.00 & -3.75 & 0.00 & 24.18 & 0.00 \\
\hline \multirow[t]{2}{*}{2013} & 0.00 & 31.59 & 0.00 & 9.59 & 0.00 & 44.72 & 0.00 & 49.96 & 0.00 & 22.44 \\
\hline & 14.63 & 0.00 & 36.67 & 0.00 & 1.54 & 0.00 & -3.70 & 0.00 & 23.82 & 0.00 \\
\hline \multirow[t]{2}{*}{2014} & 0.00 & 35.30 & 0.00 & 10.71 & 0.00 & 49.98 & 0.00 & 55.83 & 0.00 & 25.07 \\
\hline & 16.35 & 0.00 & 40.99 & 0.00 & 1.72 & 0.00 & -4.13 & 0.00 & 26.63 & 0.00 \\
\hline \multirow[t]{2}{*}{2015} & 0.00 & 36.57 & 0.00 & 11.10 & 0.00 & 51.78 & 0.00 & 57.84 & 0.00 & 25.98 \\
\hline & 16.94 & 0.00 & 42.46 & 0.00 & 1.79 & 0.00 & -4.28 & 0.00 & 27.58 & 0.00 \\
\hline \multirow[t]{2}{*}{2016} & 0.00 & 37.10 & 0.00 & 11.26 & 0.00 & 52.52 & 0.00 & 58.67 & 0.00 & 26.35 \\
\hline & 17.18 & 0.00 & 43.07 & 0.00 & 1.81 & 0.00 & -4.34 & 0.00 & 27.98 & 0.00 \\
\hline
\end{tabular}

The boundaries, based on these requirements, are shown below: 
Table 11. Boundaries (based on country)

\begin{tabular}{|c|c|c|c|c|c|c|}
\hline & & Question 1 & Question 2 & Question 3 & Question 4 & Question 5 \\
\hline \multirow{2}{*}{ Angola } & Lower & -26.38 & -8.00 & -37.34 & -41.72 & -18.73 \\
\hline & Upper & 27.17 & 68.12 & 2.86 & -6.87 & 44.25 \\
\hline \multirow{2}{*}{ Benin } & Lower & -2.29 & -0.70 & -3.24 & -3.62 & -1.63 \\
\hline & Upper & 2.36 & 5.91 & 0.25 & -0.60 & 3.84 \\
\hline \multirow{2}{*}{ Cameroon } & Lower & -7.82 & -2.37 & -11.08 & -12.38 & -5.56 \\
\hline & Upper & 8.06 & 20.21 & 0.85 & -2.04 & 13.13 \\
\hline \multirow{2}{*}{ Dem. Congo Rep. } & Lower & -5.39 & -1.64 & -7.63 & -8.52 & -3.83 \\
\hline & Upper & 5.55 & 13.92 & 0.59 & -1.40 & 9.04 \\
\hline \multirow{2}{*}{ Egypt } & Lower & -67.48 & -20.48 & -95.54 & -106.73 & -47.94 \\
\hline & Upper & 69.51 & 174.28 & 7.33 & -17.56 & 113.21 \\
\hline \multirow{2}{*}{ Equatorial Guinea } & Lower & -0.36 & -0.11 & -0.51 & -0.57 & -0.26 \\
\hline & Upper & 0.37 & 0.93 & 0.04 & -0.09 & 0.60 \\
\hline \multirow{2}{*}{ Eritrea } & Lower & -5.10 & -1.55 & -7.23 & -8.07 & -3.63 \\
\hline & Upper & 5.26 & 13.18 & 0.55 & -1.33 & 8.56 \\
\hline \multirow{2}{*}{ Ghana } & Lower & -0.27 & -0.08 & -0.38 & -0.43 & -0.19 \\
\hline & Upper & 0.28 & 0.70 & 0.03 & -0.07 & 0.45 \\
\hline \multirow{2}{*}{ Guinea } & Lower & -1.51 & -0.46 & -2.14 & -2.39 & -1.07 \\
\hline & Upper & 1.56 & 3.90 & 0.16 & -0.39 & 2.53 \\
\hline \multirow{2}{*}{ Guinea Bissau } & Lower & -11.18 & -3.39 & -15.83 & -17.69 & -7.94 \\
\hline & Upper & 11.52 & 28.88 & 1.21 & -2.91 & 18.76 \\
\hline \multirow{2}{*}{ Ivory Coast } & Lower & -8.37 & -2.54 & -11.84 & -13.23 & -5.94 \\
\hline & Upper & 8.62 & 21.60 & 0.91 & -2.18 & 14.03 \\
\hline \multirow{2}{*}{ Kenya } & Lower & -13.19 & -4.00 & -18.68 & -20.86 & -9.37 \\
\hline & Upper & 13.59 & 34.07 & 1.43 & -3.43 & 22.13 \\
\hline \multirow{2}{*}{ Liberia } & Lower & -0.42 & -0.13 & -0.60 & -0.67 & -0.30 \\
\hline & Upper & 0.44 & 1.09 & 0.05 & -0.11 & 0.71 \\
\hline \multirow{2}{*}{ Madagascar } & Lower & -2.80 & -0.85 & -3.96 & -4.42 & -1.99 \\
\hline & Upper & 2.88 & 7.22 & 0.30 & -0.73 & 4.69 \\
\hline \multirow{2}{*}{ Mauritania } & Lower & -1.44 & -0.44 & -2.04 & -2.28 & -1.03 \\
\hline & Upper & 1.49 & 3.73 & 0.16 & $\begin{array}{l}-0.38 \\
\end{array}$ & 2.42 \\
\hline
\end{tabular}




\begin{tabular}{lllllll}
\hline \multirow{2}{*}{ Morocco } & Lower & -29.86 & -9.06 & -42.27 & -47.22 & -21.21 \\
& Upper & 30.75 & 77.11 & 3.24 & -7.77 & 50.09 \\
\hline \multirow{2}{*}{ Mozambique } & Lower & -3.42 & -1.04 & -4.84 & -5.40 & -2.43 \\
& Upper & 3.52 & 8.82 & 0.37 & -0.89 & 5.73 \\
\hline \multirow{2}{*}{ Nigeria } & Lower & -117.33 & -35.60 & -166.12 & -185.57 & -83.34 \\
& Upper & 120.85 & 303.02 & 12.74 & -30.54 & 196.84 \\
\hline \multirow{2}{*}{ Seychelles } & Lower & -0.33 & -0.10 & -0.46 & -0.52 & -0.23 \\
& Upper & 0.34 & 0.85 & 0.04 & -0.09 & 0.55 \\
\multirow{2}{*}{ Sierra Leone } & Lower & -0.89 & -0.27 & -1.26 & -1.41 & -0.63 \\
& Upper & 0.92 & 2.31 & 0.10 & -0.23 & 1.50 \\
\hline \multirow{2}{*}{ Somalia } & Lower & 0.00 & 0.00 & 0.00 & 0.00 & 0.00 \\
& Upper & 0.00 & 0.00 & 0.00 & 0.00 & 0.00 \\
\hline \multirow{2}{*}{ Tanzania } & Lower & -10.58 & -3.21 & -14.98 & -16.73 & -7.51 \\
& Upper & 10.90 & 27.32 & 1.15 & -2.75 & 17.75 \\
\hline \multirow{2}{*}{ Togo } & Lower & -1.04 & -0.31 & -1.48 & -1.65 & -0.74 \\
& Upper & 1.08 & 2.70 & 0.11 & -0.27 & 1.75 \\
\hline
\end{tabular}

This is shown in the following figure:

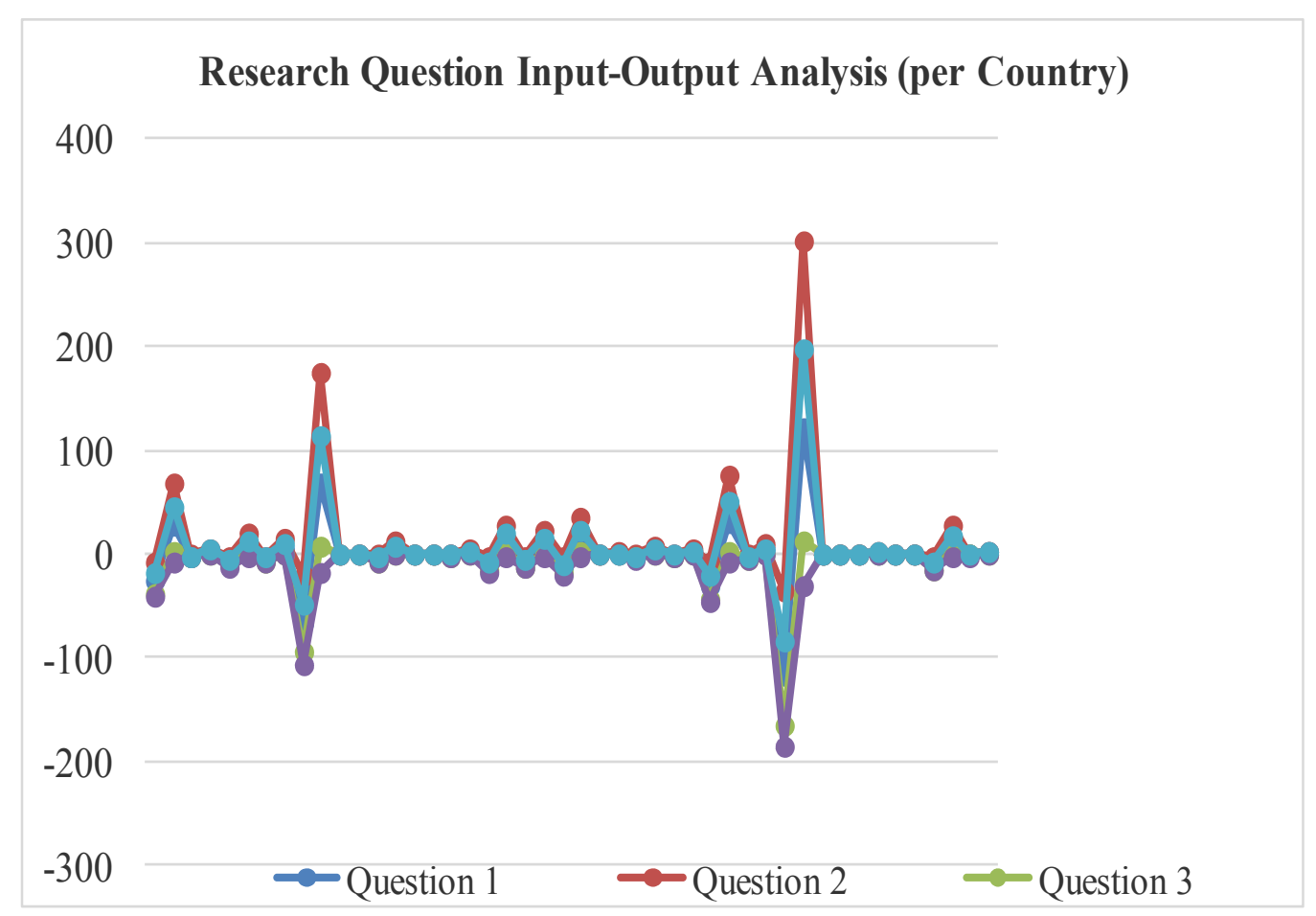

Figure 2. Input-Output analysis (per country) source: author's compilation. (2018) 
When determining the sum of all the differences, the input-output result, based on country, is 644.47 (in the comparison of average of attacks and Liner Shipping connectivity); 916.2 (in the comparison of average of attacks and growth domestic product growth rate); 483.92 (in the comparison of average of attacks and imports growth rate); 419.45 (in the comparison of average of attacks and exports as a percent of gross domestic product); and 758.06 (in the comparison of average of attacks and oil production as oil rents as a percent of gross domestic product).

The boundaries for the years are shown below:

Table 12. Boundaries (based on Year)

\begin{tabular}{|c|c|c|c|c|c|c|}
\hline & & Question 1 & Question 2 & Question 3 & Question 4 & Question 5 \\
\hline \multirow{2}{*}{2007} & Lower & -11.21 & -3.40 & -15.88 & -17.74 & -7.97 \\
\hline & Upper & 11.55 & 28.96 & 1.22 & -2.92 & 18.81 \\
\hline \multirow{2}{*}{2008} & Lower & -11.97 & -3.63 & -16.95 & -18.94 & -8.51 \\
\hline & Upper & 12.33 & 30.92 & 1.30 & -3.12 & 20.09 \\
\hline \multirow{2}{*}{2009} & Lower & -9.49 & -2.88 & -13.43 & -15.01 & -6.74 \\
\hline & Upper & 9.77 & 24.51 & 1.03 & -2.47 & 15.92 \\
\hline \multirow{2}{*}{2010} & Lower & -13.26 & -4.02 & -18.78 & -20.97 & -9.42 \\
\hline & Upper & 13.66 & 34.25 & 1.44 & -3.45 & 22.25 \\
\hline \multirow{2}{*}{2011} & Lower & -13.85 & -4.20 & -19.61 & -21.91 & -9.84 \\
\hline & Upper & 14.27 & 35.77 & 1.50 & -3.61 & 23.24 \\
\hline \multirow{2}{*}{2012} & Lower & -14.41 & -4.37 & -20.41 & -22.80 & -10.24 \\
\hline & Upper & 14.85 & 37.23 & 1.57 & -3.75 & 24.18 \\
\hline \multirow{2}{*}{2013} & Lower & -14.20 & -4.31 & -20.11 & -22.46 & -10.09 \\
\hline & Upper & 14.63 & 36.67 & 1.54 & -3.70 & 23.82 \\
\hline \multirow{2}{*}{2014} & Lower & -15.87 & -4.82 & -22.47 & -25.10 & -11.27 \\
\hline & Upper & 16.35 & 40.99 & 1.72 & -4.13 & 26.63 \\
\hline \multirow{2}{*}{2015} & Lower & -16.44 & -4.99 & -23.28 & -26.00 & -11.68 \\
\hline & Upper & 16.94 & 42.46 & 1.79 & -4.28 & 27.58 \\
\hline \multirow{2}{*}{2016} & Lower & -16.68 & -5.06 & -23.61 & -26.38 & -11.85 \\
\hline & Upper & 17.18 & 43.07 & 1.81 & -4.34 & 27.98 \\
\hline
\end{tabular}

This is shown in the following figure: 


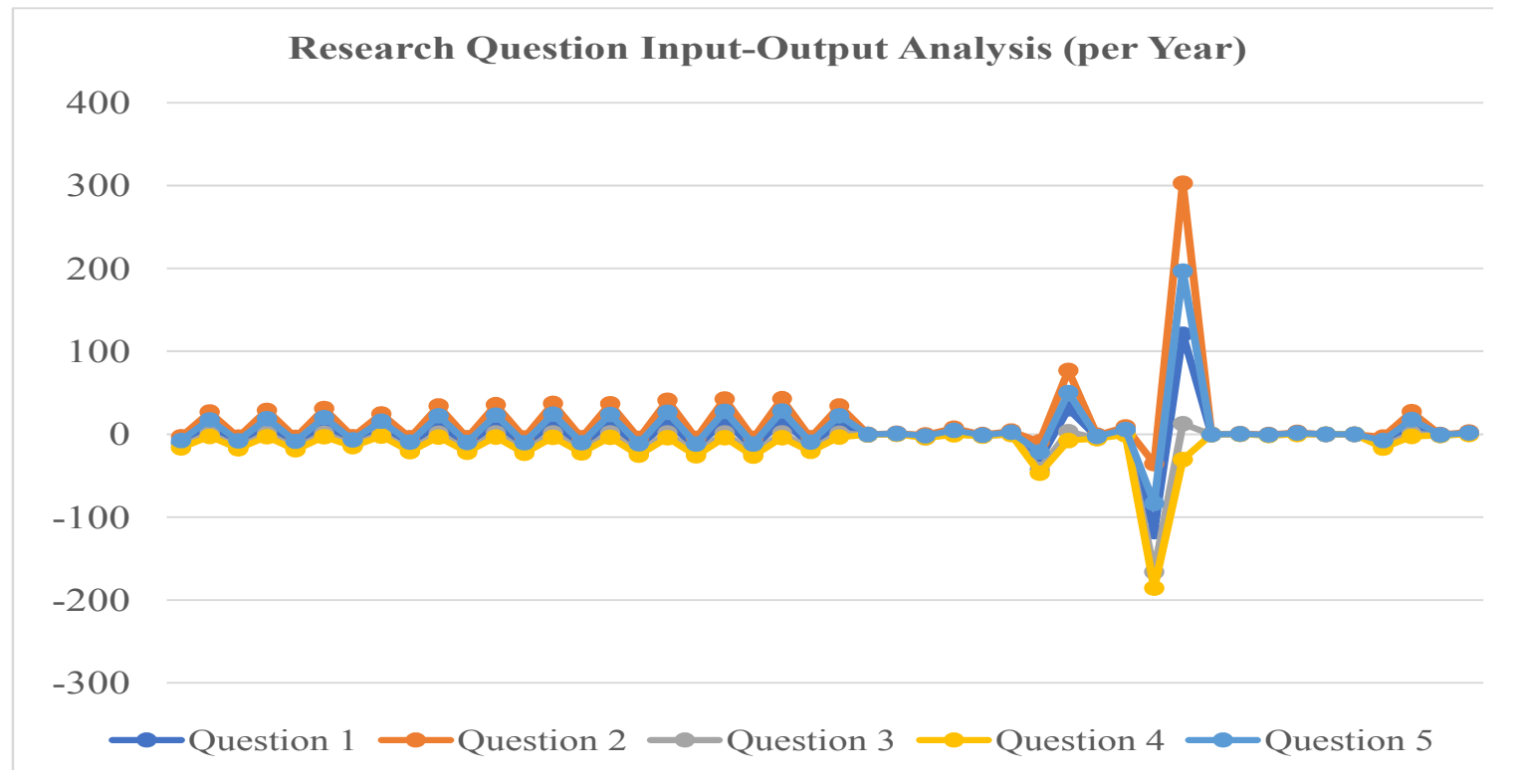

Figure 3. Input-Output analysis (per year) source: author's compilation. (2018)

When determining the sum of all the differences, the input-output result, based on years, is 300.122 (in the comparison of average of attacks and Liner Shipping connectivity); 392.95 (in the comparison of average of attacks and growth domestic product growth rate); 207.54 (in the comparison of average of attacks and imports growth rate); 179.91 (in the comparison of average of attacks and exports as a percent of gross domestic product); and 325.12 (in the comparison of average of attacks and oil production as oil rents as a percent of gross domestic product). This comparison is shown below:

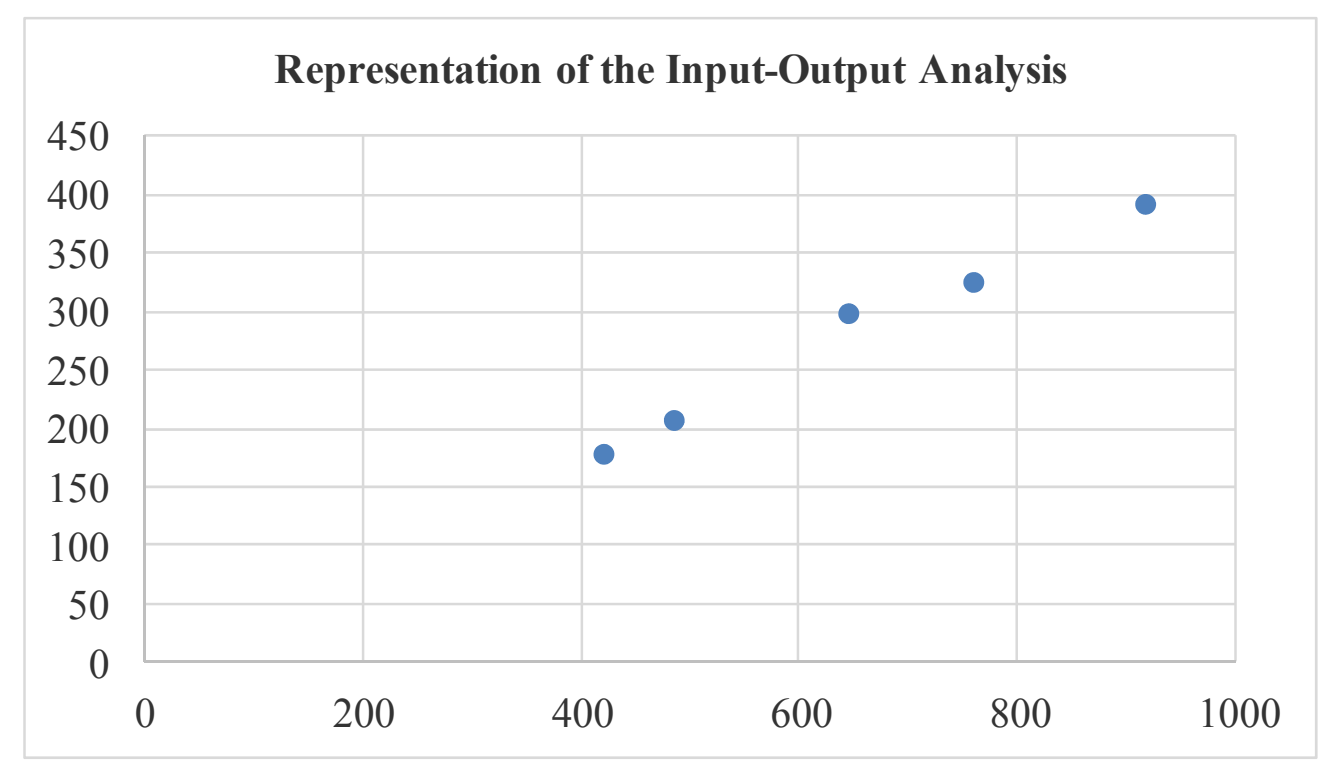

Figure 4. Representation of the Input-Output analysis source: author's compilation (2018) 


\subsection{Fixed Effects Model}

The following table shows the results of the fixed effects model:

Table 13. Fixed effects model

\begin{tabular}{|c|c|c|c|c|}
\hline Country & Distance & Total distance & Piracy attacks & \\
\hline Angola & 2,280 & 57,953 & 0.70 & 0.0275 \\
\hline Benin & 639 & 57,953 & 2.40 & 0.0265 \\
\hline Cameroon & 1,034 & 57,953 & 1.30 & 0.0232 \\
\hline Dem. Congo Rep. & 1,412 & 57,953 & 4.50 & 0.1096 \\
\hline Egypt & 3,853 & 57,953 & 2.20 & 0.1463 \\
\hline Equatorial Guinea & 760 & 57,953 & 0.10 & 0.0013 \\
\hline Eritrea & 4,141 & 57,953 & 0.10 & 0.0071 \\
\hline Ghana & 705 & 57,953 & 2.50 & 0.0304 \\
\hline Guinea & 1,635 & 57,953 & 2.80 & 0.0790 \\
\hline Guinea Bissau & 2,266 & 57,953 & 0.10 & 0.0039 \\
\hline Ivory Coast & 1,111 & 57,953 & 2.40 & 0.0460 \\
\hline Kenya & 3,817 & 57,953 & 1.40 & 0.0922 \\
\hline Liberia & 1,488 & 57,953 & 0.60 & 0.0154 \\
\hline Madagascar & 5,338 & 57,953 & 0.10 & 0.0092 \\
\hline Mauritania & 2,490 & 57,953 & 0.10 & 0.0043 \\
\hline Morocco & 3,317 & 57,953 & 0.50 & 0.0286 \\
\hline Mozambique & 4,277 & 57,953 & 1.20 & 0.0886 \\
\hline Nigeria & 806 & 57,953 & 26.60 & 0.3699 \\
\hline Seychelles & 5,821 & 57,953 & 0.10 & 0.0100 \\
\hline Sierra Leone & 1,793 & 57,953 & 0.70 & 0.0217 \\
\hline Somalia & 4,706 & 57,953 & 48.90 & 3.9709 \\
\hline Tanzania & 3,633 & 57,953 & 3.50 & 0.2194 \\
\hline Togo & 631 & 57,953 & 3.40 & 0.0370 \\
\hline
\end{tabular}

This is shown in the Figure 5: 


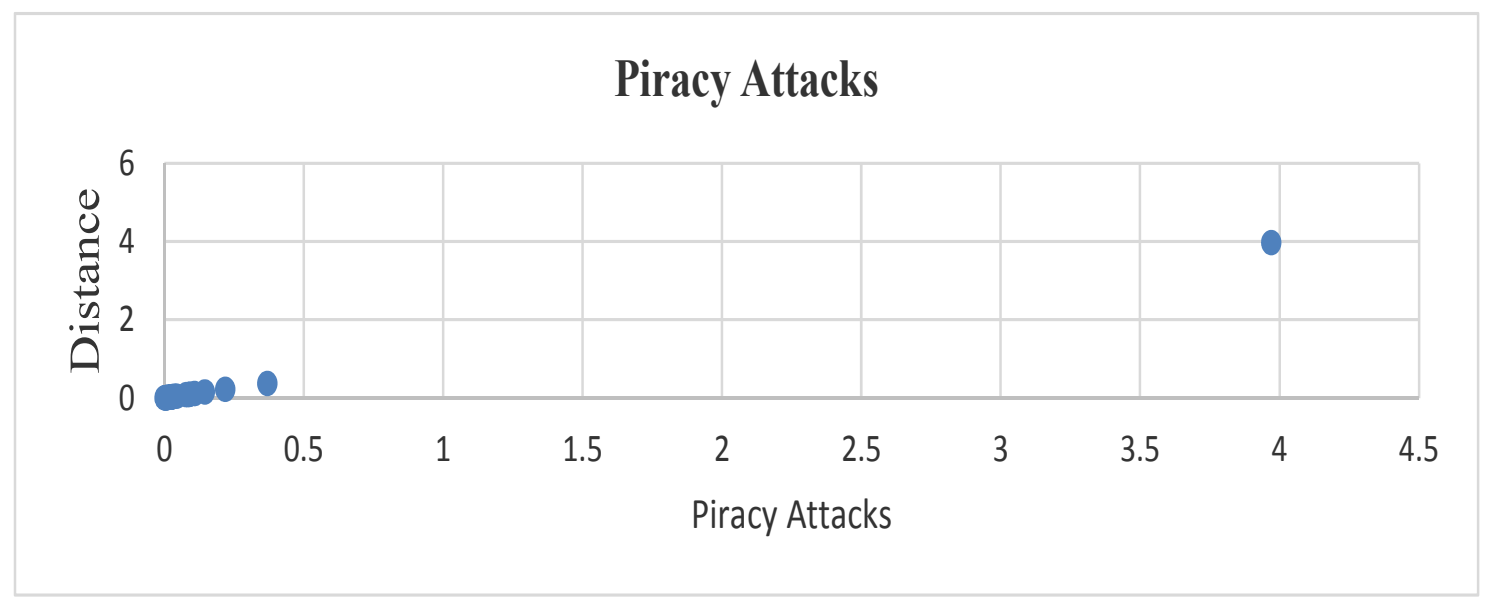

Figure 5. Piracy attacks based on distance source: author's compilation. (2018)

\section{Correlation Analysis}

The correlation analysis will occur in respect to the countries and years and based on the research questions. The results are shown as follows:

Table 14. Correlation analysis by country (Question 1)

\begin{tabular}{llllll}
\hline & $\mathbf{X}$ & $\mathbf{Y}$ & $\mathbf{X Y}$ & $\mathbf{X}^{\mathbf{2}}$ & $\mathbf{Y}^{\mathbf{2}}$ \\
\hline Angola & 0.70 & 14.97 & 10.48 & 0.49 & 224.10 \\
Benin & 2.40 & 14.34 & 34.42 & 5.76 & 205.64 \\
Cameroon & 1.30 & 12.00 & 15.60 & 1.69 & 144.00 \\
Dem. Congo Rep. & 4.50 & 9.49 & 42.71 & 20.25 & 90.06 \\
Egypt & 2.20 & 54.92 & 120.82 & 4.84 & $3,016.21$ \\
Equatorial Guinea & 0.10 & 5.41 & 0.54 & 0.01 & 29.27 \\
Eritrea & 0.10 & 2.98 & 0.30 & 0.01 & 8.88 \\
Ghana & 2.50 & 18.92 & 47.30 & 6.25 & 357.97 \\
Guinea & 2.80 & 7.49 & 20.97 & 7.84 & 56.10 \\
Guinea Bissau & 0.10 & 4.19 & 0.42 & 0.01 & 17.56 \\
Ivory Coast & 2.40 & 19.54 & 46.90 & 5.76 & 381.81 \\
Kenya & 1.40 & 11.98 & 16.77 & 1.96 & 143.52 \\
Liberia & 0.60 & 6.26 & 3.76 & 0.36 & 39.19 \\
Madagascar & 0.10 & 9.66 & 0.97 & 0.01 & 93.32 \\
Mauritania & 0.10 & 7.02 & 0.70 & 0.01 & 49.28 \\
\hline
\end{tabular}




\begin{tabular}{llllll}
\hline Morocco & 0.50 & 48.96 & 24.48 & 0.25 & $2,397.08$ \\
Mozambique & 1.20 & 9.30 & 11.16 & 1.44 & 86.49 \\
Nigeria & 26.60 & 21.07 & 560.46 & 707.56 & 443.94 \\
Seychelles & 0.10 & 6.49 & 0.65 & 0.01 & 42.12 \\
Sierra Leone & 0.70 & 6.07 & 4.25 & 0.49 & 36.84 \\
Somalia & 48.90 & 4.35 & 212.72 & $2,391.21$ & 18.92 \\
Tanzania & 3.50 & 6.40 & 22.40 & 12.25 & 40.96 \\
Togo & 3.40 & 3.57 & 12.14 & 11.56 & 12.74 \\
Totals & 106.20 & 305.38 & $1,210.90$ & $3,180.02$ & $7,936.00$ \\
$\mathrm{~N}$ & 23 & & & & \\
Numerator & $-4,580.68$ & & & & \\
Denominator & $215,333.31$ & & & & \\
$\mathrm{r}$ & -0.0213 & & & & \\
\hline
\end{tabular}

Table 15. Correlation analysis by year (Question 1)

\begin{tabular}{llllll}
\hline & $\mathbf{X}$ & $\mathbf{Y}$ & $\mathbf{X Y}$ & $\mathbf{X}^{\mathbf{2}}$ & $\mathbf{Y}^{\mathbf{2}}$ \\
\hline $\mathbf{2 0 0 7}$ & 10.83 & 407.84 & 10.83 & 117.29 & $166,333.47$ \\
$\mathbf{2 0 0 8}$ & 9.26 & 407.73 & 9.26 & 85.75 & $166,243.75$ \\
$\mathbf{2 0 0 9}$ & 18.07 & 407.77 & 18.07 & 326.52 & $166,276.37$ \\
$\mathbf{2 0 1 0}$ & 30.2 & 408.07 & 30.20 & 912.04 & $166,521.12$ \\
$\mathbf{2 0 1 1}$ & 34.69 & 408.14 & 34.69 & $1,203.40$ & $166,578.26$ \\
$\mathbf{2 0 1 2}$ & 11.68 & 408.12 & 11.68 & 136.42 & $166,561.93$ \\
$\mathbf{2 0 1 3}$ & 6.78 & 408.38 & 6.78 & 45.97 & $166,774.22$ \\
$\mathbf{2 0 1 4}$ & 4.15 & 408.31 & 4.15 & 17.22 & $166,717.06$ \\
$\mathbf{2 0 1 5}$ & 3.04 & 408.26 & 3.04 & 9.24 & $166,676.23$ \\
$\mathbf{2 0 1 6}$ & 7.83 & 408.50 & 7.83 & 61.31 & $166,872.25$ \\
Totals & 136.53 & $4,081.12$ & 136.53 & $2,915.16$ & $1,665,554.67$ \\
N & 10 & & & & \\
Numerator & $-555,830.01$ & & & & \\
Denominator & $1,488,338.72$ & & & & \\
r & -0.3735 & & & & \\
\hline
\end{tabular}

For the first research question, there is a negative correlation for both the individual countries 
and years, suggesting that there is a negative relationship between piracy attacks and the Liner Shipping index.

Table 16. Correlation analysis by country (Question 2)

\begin{tabular}{|c|c|c|c|c|c|}
\hline & $\mathbf{X}$ & $\mathbf{Y}$ & $\mathbf{X Y}$ & $\mathrm{X}^{2}$ & $\mathbf{Y}^{2}$ \\
\hline Angola & 0.70 & 6.59 & 4.61 & 0.49 & 43.43 \\
\hline Benin & 2.40 & 4.27 & 10.25 & 5.76 & 18.23 \\
\hline Cameroon & 1.30 & 4.19 & 5.45 & 1.69 & 17.56 \\
\hline Dem. Congo Rep. & 4.50 & 5.10 & 22.95 & 20.25 & 26.01 \\
\hline Egypt & 2.20 & 4.19 & 9.22 & 4.84 & 17.56 \\
\hline Equatorial Guinea & 0.10 & 1.67 & 0.17 & 0.01 & 2.79 \\
\hline Eritrea & 0.10 & 0.64 & 0.06 & 0.01 & 0.41 \\
\hline Ghana & 2.50 & 6.84 & 17.10 & 6.25 & 46.79 \\
\hline Guinea & 2.80 & 2.42 & 6.78 & 7.84 & 5.86 \\
\hline Guinea Bissau & 0.10 & 3.54 & 0.35 & 0.01 & 12.53 \\
\hline Ivory Coast & 2.40 & 5.15 & 12.36 & 5.76 & 26.52 \\
\hline Kenya & 1.40 & 5.23 & 7.32 & 1.96 & 27.35 \\
\hline Liberia & 0.60 & 5.21 & 3.13 & 0.36 & 27.14 \\
\hline Madagascar & 0.10 & 2.69 & 0.27 & 0.01 & 7.24 \\
\hline Mauritania & 0.10 & 3.32 & 0.33 & 0.01 & 11.02 \\
\hline Morocco & 0.50 & 3.85 & 1.93 & 0.25 & 14.82 \\
\hline Mozambique & 1.20 & 6.67 & 8.00 & 1.44 & 44.49 \\
\hline Nigeria & 26.60 & 4.99 & 132.73 & 707.56 & 24.90 \\
\hline Seychelles & 0.10 & 4.50 & 0.45 & 0.01 & 20.25 \\
\hline Sierra Leone & 0.70 & 5.43 & 3.80 & 0.49 & 29.48 \\
\hline Somalia & 48.90 & 0.00 & 0.00 & $2,391.21$ & 0.00 \\
\hline Tanzania & 3.50 & 6.70 & 23.45 & 12.25 & 44.89 \\
\hline Togo & 3.40 & 4.19 & 14.25 & 11.56 & 17.56 \\
\hline Totals & 106.20 & 97.38 & 284.96 & $3,180.02$ & 486.83 \\
\hline $\mathrm{N}$ & 23 & & & & \\
\hline Numerator & $-3,787.77$ & & & & \\
\hline Denominator & $51,104.73$ & & & & \\
\hline $\mathrm{r}$ & -0.0741 & & & & \\
\hline
\end{tabular}


Table 17. Correlation analysis by year (Question 2)

\begin{tabular}{llllll}
\hline & $\mathbf{X}$ & $\mathbf{Y}$ & $\mathbf{X Y}$ & $\mathbf{X}^{2}$ & $\mathbf{Y}^{2}$ \\
\hline 2007 & 10.83 & 5.05 & 10.83 & 117.29 & 25.50 \\
2008 & 9.26 & 5.36 & 9.26 & 85.75 & 28.73 \\
2009 & 18.07 & 2.70 & 18.07 & 326.52 & 7.29 \\
2010 & 30.2 & 3.71 & 30.20 & 912.04 & 13.76 \\
2011 & 34.69 & 3.64 & 34.69 & $1,203.40$ & 13.25 \\
2012 & 11.68 & 3.67 & 11.68 & 136.42 & 13.47 \\
2013 & 6.78 & 4.66 & 6.78 & 45.97 & 21.72 \\
2014 & 4.15 & 2.76 & 4.15 & 17.22 & 7.62 \\
2015 & 3.04 & 6.10 & 3.04 & 9.24 & 37.21 \\
2016 & 7.83 & 3.88 & 7.83 & 61.31 & 15.05 \\
Totals & 136.53 & 41.53 & 136.53 & $2,915.16$ & 183.60 \\
N & 10 & & & & \\
Numerator & $-4,304.79$ & & & & \\
Denominator & $15,663.87$ & & & & \\
r & -0.2748 & & & & \\
\hline
\end{tabular}

For the second research question, there is a negative correlation for both the individual countries and years, suggesting that there is a negative relationship between piracy attacks and the gross domestic product growth rate.

Table 18. Correlation analysis by country (Question 3)

\begin{tabular}{llllll}
\hline & $\mathbf{X}$ & $\mathbf{Y}$ & $\mathbf{X Y}$ & $\mathbf{X}^{2}$ & $\mathbf{Y}^{2}$ \\
\hline Angola & 0.70 & 0.00 & 0.00 & 0.49 & 0.00 \\
Benin & 2.40 & 9.30 & 22.32 & 5.76 & 86.49 \\
Cameroon & 1.30 & 9.03 & 11.74 & 1.69 & 81.54 \\
Dem. Congo Rep. & 4.50 & 3.66 & 16.47 & 20.25 & 13.40 \\
Egypt & 2.20 & 5.03 & 11.07 & 4.84 & 25.30 \\
Equatorial Guinea & 0.10 & 2.95 & 0.30 & 0.01 & 8.70 \\
Eritrea & 0.10 & 0.26 & 0.03 & 0.01 & 0.07 \\
Ghana & 2.50 & 11.65 & 29.13 & 6.25 & 135.72 \\
Guinea & 2.80 & 7.58 & 21.22 & 7.84 & 57.46 \\
\hline
\end{tabular}




\begin{tabular}{|c|c|c|c|c|c|}
\hline Guinea Bissau & 0.10 & 6.82 & 0.68 & 0.01 & 46.51 \\
\hline Ivory Coast & 2.40 & 5.17 & 12.41 & 5.76 & 26.73 \\
\hline Kenya & 1.40 & 5.85 & 8.19 & 1.96 & 34.22 \\
\hline Liberia & 0.60 & 2.40 & 1.44 & 0.36 & 5.76 \\
\hline Madagascar & 0.10 & 5.91 & 0.59 & 0.01 & 34.93 \\
\hline Mauritania & 0.10 & 6.45 & 0.65 & 0.01 & 41.60 \\
\hline Morocco & 0.50 & 5.10 & 2.55 & 0.25 & 26.01 \\
\hline Mozambique & 1.20 & 17.59 & 21.11 & 1.44 & 309.41 \\
\hline Nigeria & 26.60 & 4.92 & 130.87 & 707.56 & 24.21 \\
\hline Seychelles & 0.10 & 0.00 & 0.00 & 0.01 & 0.00 \\
\hline Sierra Leone & 0.70 & 19.47 & 13.63 & 0.49 & 379.08 \\
\hline Somalia & 48.90 & 0.00 & 0.00 & $2,391.21$ & 0.00 \\
\hline Tanzania & 3.50 & 9.99 & 34.97 & 12.25 & 99.80 \\
\hline Togo & 3.40 & 18.82 & 63.99 & 11.56 & 354.19 \\
\hline Totals & 106.20 & 157.95 & 403.33 & $3,180.02$ & $1,791.13$ \\
\hline $\mathrm{N}$ & \multicolumn{5}{|l|}{23} \\
\hline Numerator & \multicolumn{5}{|c|}{$-7,497.63$} \\
\hline Denominator & \multicolumn{5}{|c|}{$101,113.38$} \\
\hline $\mathrm{r}$ & \multicolumn{5}{|c|}{-0.0742} \\
\hline
\end{tabular}

Table 19. Correlation analysis by year (Question 3)

\begin{tabular}{llllll}
\hline & $\mathbf{X}$ & $\mathbf{Y}$ & $\mathbf{X Y}$ & $\mathbf{X}^{\mathbf{2}}$ & $\mathbf{Y}^{\mathbf{2}}$ \\
\hline 2007 & 10.83 & 23.02 & 10.83 & 117.29 & 529.92 \\
2008 & 9.26 & 11.23 & 9.26 & 85.75 & 126.11 \\
2009 & 18.07 & 15.57 & 18.07 & 326.52 & 242.42 \\
2010 & 30.2 & 11.05 & 30.20 & 912.04 & 122.10 \\
2011 & 34.69 & 23.04 & 34.69 & $1,203.40$ & 530.84 \\
2012 & 11.68 & 22.97 & 11.68 & 136.42 & 527.62 \\
2013 & 6.78 & 19.59 & 6.78 & 45.97 & 383.77 \\
2014 & 4.15 & 10.60 & 4.15 & 17.22 & 112.36 \\
2015 & 3.04 & 14.04 & 3.04 & 9.24 & 197.12 \\
2016 & 7.83 & 9.39 & 7.83 & 61.31 & 88.17 \\
Totals & 136.53 & 160.50 & 136.53 & $2,915.16$ & $2,860.45$ \\
\hline
\end{tabular}




\begin{tabular}{ll}
\hline $\mathrm{N}$ & 10 \\
Numerator & $-20,547.77$ \\
Denominator & $61,921.13$ \\
$\mathrm{r}$ & -0.3318 \\
\hline
\end{tabular}

For the third research question, there is a negative correlation in both countries and years, suggesting that there is a negative relationship between piracy attacks and the imports growth rate.

Table 20. Correlation analysis by country (Question 4)

\begin{tabular}{|c|c|c|c|c|c|}
\hline & $\mathbf{X}$ & $\mathbf{Y}$ & $\mathbf{X Y}$ & $\mathrm{X}^{2}$ & $\mathbf{Y}^{2}$ \\
\hline Angola & 0.70 & 56.59 & 39.61 & 0.49 & $3,202.43$ \\
\hline Benin & 2.40 & 25.46 & 61.10 & 5.76 & 648.21 \\
\hline Cameroon & 1.30 & 20.34 & 26.44 & 1.69 & 413.72 \\
\hline Dem. Congo Rep. & 4.50 & 55.51 & 249.80 & 20.25 & $3,081.36$ \\
\hline Egypt & 2.20 & 20.14 & 44.31 & 4.84 & 405.62 \\
\hline Equatorial Guinea & 0.10 & 73.44 & 7.34 & 0.01 & $5,393.43$ \\
\hline Eritrea & 0.10 & 3.39 & 0.34 & 0.01 & 11.49 \\
\hline Ghana & 2.50 & 34.39 & 85.98 & 6.25 & $1,182.67$ \\
\hline Guinea & 2.80 & 28.56 & 79.97 & 7.84 & 815.67 \\
\hline Guinea Bissau & 0.10 & 21.53 & 2.15 & 0.01 & 463.54 \\
\hline Ivory Coast & 2.40 & 44.96 & 107.90 & 5.76 & $2,021.40$ \\
\hline Kenya & 1.40 & 19.86 & 27.80 & 1.96 & 394.42 \\
\hline Liberia & 0.60 & 30.24 & 18.14 & 0.36 & 914.46 \\
\hline Madagascar & 0.10 & 28.80 & 2.88 & 0.01 & 829.44 \\
\hline Mauritania & 0.10 & 45.48 & 4.55 & 0.01 & $2,068.43$ \\
\hline Morocco & 0.50 & 33.63 & 16.82 & 0.25 & $1,130.98$ \\
\hline Mozambique & 1.20 & 31.82 & 38.18 & 1.44 & $1,012.51$ \\
\hline Nigeria & 26.60 & 23.95 & 637.07 & 707.56 & 573.60 \\
\hline Seychelles & 0.10 & 75.09 & 7.51 & 0.01 & $5,638.51$ \\
\hline Sierra Leone & 0.70 & 21.04 & 14.73 & 0.49 & 442.68 \\
\hline Somalia & 48.90 & 5.84 & 285.58 & $2,391.21$ & 34.11 \\
\hline Tanzania & 3.50 & 19.20 & 67.20 & 12.25 & 368.64 \\
\hline
\end{tabular}




\begin{tabular}{llllll}
\hline Togo & 3.40 & 41.86 & 142.32 & 11.56 & $1,752.26$ \\
Totals & 106.20 & 761.12 & $1,967.73$ & $3,180.02$ & $32,799.58$ \\
$\mathrm{~N}$ & 23 & & & & \\
Numerator & $-35,573.22$ & & & & \\
Denominator & $423,849.09$ & & & \\
$\mathrm{r}$ & -0.0839 & & & & \\
\hline
\end{tabular}

Table 21. Correlation analysis by year (Question 4)

\begin{tabular}{llllll}
\hline & $\mathbf{X}$ & $\mathbf{Y}$ & $\mathbf{X Y}$ & $\mathbf{X}^{2}$ & $\mathbf{Y}^{2}$ \\
\hline 2007 & 10.83 & 24.67 & 10.83 & 117.29 & 608.61 \\
2008 & 9.26 & 24.82 & 9.26 & 85.75 & 616.03 \\
2009 & 18.07 & 23.71 & 18.07 & 326.52 & 562.16 \\
2010 & 30.2 & 23.81 & 30.20 & 912.04 & 566.92 \\
2011 & 34.69 & 22.77 & 34.69 & $1,203.40$ & 518.47 \\
2012 & 11.68 & 22.07 & 11.68 & 136.42 & 487.08 \\
2013 & 6.78 & 19.58 & 6.78 & 45.97 & 383.38 \\
2014 & 4.15 & 18.23 & 4.15 & 17.22 & 332.33 \\
2015 & 3.04 & 14.97 & 3.04 & 9.24 & 224.10 \\
2016 & 7.83 & 14.55 & 7.83 & 61.31 & 211.70 \\
Totals & 136.53 & 209.18 & 136.53 & $2,915.16$ & $4,510.79$ \\
$\mathrm{~N}$ & 10 & & & & \\
Numerator & $-27,194.05$ & & & & \\
Denominator & $77,546.53$ & & & & \\
$\mathrm{r}$ & -0.3507 & & & &
\end{tabular}

For the fourth research question, there is a negative correlation in relation to both country and year, suggesting that there is a negative relationship between piracy attacks and exports as a percentage of gross domestic product. 
Table 22. Correlation analysis by country (Question 5)

\begin{tabular}{|c|c|c|c|c|c|}
\hline & $\mathbf{X}$ & $\mathbf{Y}$ & $\mathbf{X Y}$ & $X^{2}$ & $\mathbf{Y}^{2}$ \\
\hline Angola & 0.70 & 33.24 & 23.27 & 0.49 & $1,104.90$ \\
\hline Benin & 2.40 & 0.00 & 0.00 & 5.76 & 0.00 \\
\hline Cameroon & 1.30 & 5.06 & 6.58 & 1.69 & 25.60 \\
\hline Dem. Congo Rep. & 4.50 & 20.06 & 90.27 & 20.25 & 402.40 \\
\hline Egypt & 2.20 & 6.93 & 15.25 & 4.84 & 48.02 \\
\hline Equatorial Guinea & 0.10 & 28.70 & 2.87 & 0.01 & 823.69 \\
\hline Eritrea & 0.10 & 0.00 & 0.00 & 0.01 & 0.00 \\
\hline Ghana & 2.50 & 2.36 & 5.90 & 6.25 & 5.57 \\
\hline Guinea & 2.80 & 0.00 & 0.00 & 7.84 & 0.00 \\
\hline Guinea Bissau & 0.10 & 0.00 & 0.00 & 0.01 & 0.00 \\
\hline Ivory Coast & 2.40 & 2.44 & 5.86 & 5.76 & 5.95 \\
\hline Kenya & 1.40 & 0.00 & 0.00 & 1.96 & 0.00 \\
\hline Liberia & 0.60 & 0.00 & 0.00 & 0.36 & 0.00 \\
\hline Madagascar & 0.10 & 0.00 & 0.00 & 0.01 & 0.00 \\
\hline Mauritania & 0.10 & 3.42 & 0.34 & 0.01 & 11.70 \\
\hline Morocco & 0.50 & 0.01 & 0.01 & 0.25 & 0.00 \\
\hline Mozambique & 1.20 & 0.10 & 0.12 & 1.44 & 0.01 \\
\hline Nigeria & 26.60 & 13.37 & 355.64 & 707.56 & 178.76 \\
\hline Seychelles & 0.10 & 0.00 & 0.00 & 0.01 & 0.00 \\
\hline Sierra Leone & 0.70 & 0.00 & 0.00 & 0.49 & 0.00 \\
\hline Somalia & 48.90 & 0.00 & 0.00 & $2,391.21$ & 0.00 \\
\hline Tanzania & 3.50 & 0.00 & 0.00 & 12.25 & 0.00 \\
\hline Togo & 3.40 & 0.00 & 0.00 & 11.56 & 0.00 \\
\hline Totals & 106.20 & 115.69 & 506.10 & $3,180.02$ & $2,606.61$ \\
\hline $\mathrm{N}$ & 23 & & & & \\
\hline Numerator & -646.05 & & & & \\
\hline Denominator & $127,658.81$ & & & & \\
\hline $\mathrm{r}$ & -0.0051 & & & & \\
\hline
\end{tabular}


Table 23. Correlation analysis by year (Question 5)

\begin{tabular}{llllll}
\hline & $\mathbf{X}$ & $\mathbf{Y}$ & $\mathbf{X Y}$ & $\mathbf{X}^{2}$ & $\mathbf{Y}^{2}$ \\
\hline 2007 & 10.83 & 15.13 & 10.83 & 117.29 & 228.92 \\
2008 & 9.26 & 15.78 & 9.26 & 85.75 & 249.01 \\
2009 & 18.07 & 8.20 & 18.07 & 326.52 & 67.24 \\
2010 & 30.2 & 10.83 & 30.20 & 912.04 & 117.29 \\
2011 & 34.69 & 12.47 & 34.69 & $1,203.40$ & 155.50 \\
2012 & 11.68 & 11.88 & 11.68 & 136.42 & 141.13 \\
2013 & 6.78 & 10.03 & 6.78 & 45.97 & 100.60 \\
2014 & 4.15 & 8.35 & 4.15 & 17.22 & 69.72 \\
2015 & 3.04 & 4.01 & 3.04 & 9.24 & 16.08 \\
2016 & 7.83 & 0.00 & 7.83 & 61.31 & 0.00 \\
Totals & 136.53 & 96.68 & 136.53 & $2,915.16$ & $1,145.49$ \\
N & 10 & & & & \\
Numerator & $-11,834.42$ & & & & \\
Denominator & $39,314.60$ & & & & \\
r & -0.3010 & & & & \\
\hline
\end{tabular}

For the final research question, there is a negative correlation in relation to both country and year, suggesting that there is a negative relationship between piracy attacks and oil production as oil rents as a percentage of gross domestic product.

\section{Chi Square Test}

The chi square test results are shown below:

Table 24. Chi square test for research question 1 (by country)

\begin{tabular}{lllllll}
\hline Piracy & \multicolumn{7}{c}{ Variable Y } & & \\
\hline Observed & Expected & Observed & Expected & Total & & \\
\hline 0.70 & 4.04 & 14.97 & 11.63 & 15.67 & 2.76 & 0.75 \\
2.40 & 4.32 & 14.34 & 12.42 & 16.74 & 0.85 & 0.26 \\
1.30 & 3.43 & 12.00 & 9.87 & 13.30 & 1.32 & 0.38 \\
4.50 & 3.61 & 9.49 & 10.38 & 13.99 & 0.22 & 0.08 \\
2.20 & 14.74 & 54.92 & 42.38 & 57.12 & 10.67 & 2.86 \\
0.10 & 1.42 & 5.41 & 4.09 & 5.51 & 1.23 & 0.32 \\
\hline
\end{tabular}




\begin{tabular}{|c|c|c|c|c|c|c|}
\hline 0.10 & 0.79 & 2.98 & 2.29 & 3.08 & 0.61 & 0.16 \\
\hline 2.50 & 5.53 & 18.92 & 15.89 & 21.42 & 1.66 & 0.48 \\
\hline 2.80 & 2.66 & 7.49 & 7.63 & 10.29 & 0.01 & 0.00 \\
\hline 0.10 & 1.11 & 4.19 & 3.18 & 4.29 & 0.92 & 0.24 \\
\hline 2.40 & 5.66 & 19.54 & 16.28 & 21.94 & 1.88 & 0.54 \\
\hline 1.40 & 3.45 & 11.98 & 9.93 & 13.38 & 1.22 & 0.35 \\
\hline 0.60 & 1.77 & 6.26 & 5.09 & 6.86 & 0.77 & 0.22 \\
\hline 0.10 & 2.52 & 9.66 & 7.24 & 9.76 & 2.32 & 0.61 \\
\hline 0.10 & 1.84 & 7.02 & 5.28 & 7.12 & 1.64 & 0.43 \\
\hline 0.50 & 12.76 & 48.96 & 36.70 & 49.46 & 11.78 & 3.07 \\
\hline 1.20 & 2.71 & 9.30 & 7.79 & 10.50 & 0.84 & 0.24 \\
\hline 26.60 & 12.30 & 21.07 & 35.37 & 47.67 & 16.62 & 9.70 \\
\hline 0.10 & 1.70 & 6.49 & 4.89 & 6.59 & 1.51 & 0.39 \\
\hline 0.70 & 1.75 & 6.07 & 5.02 & 6.77 & 0.63 & 0.18 \\
\hline 48.90 & 13.74 & 4.35 & 39.51 & 53.25 & 89.97 & 284.19 \\
\hline 3.50 & 2.55 & 6.40 & 7.35 & 9.90 & 0.35 & 0.14 \\
\hline 3.40 & 1.80 & 3.57 & 5.17 & 6.97 & 1.43 & 0.72 \\
\hline 106.20 & & 305.38 & & 411.58 & 457.55 & \\
\hline Df & 21.00 & & & & & \\
\hline Alpha & 0.05 & & & & & \\
\hline Critical Value & 32.67 & & & & & \\
\hline
\end{tabular}

Table 25. Chi square test for research question 1 (by year)

\begin{tabular}{lllllll}
\hline Piracy & \multicolumn{7}{c}{ Variable Y } & & \\
\hline Observed & Expected & Observed & Expected & Total & & \\
\hline 4.65 & 3.64 & 9.44 & 10.45 & 14.09 & 0.28 & 0.11 \\
4.26 & 4.00 & 11.25 & 11.51 & 15.51 & 0.02 & 0.01 \\
5.83 & 4.64 & 12.15 & 13.34 & 17.98 & 0.31 & 0.12 \\
7.87 & 5.07 & 11.79 & 14.59 & 19.66 & 1.54 & 0.66 \\
9.43 & 5.69 & 12.60 & 16.34 & 22.03 & 2.47 & 1.11 \\
5.35 & 4.94 & 13.80 & 14.21 & 19.15 & 0.03 & 0.01 \\
3.00 & 4.28 & 13.58 & 12.30 & 16.58 & 0.38 & 0.12 \\
1.96 & 4.43 & 15.22 & 12.75 & 17.18 & 1.38 & 0.40 \\
\hline
\end{tabular}




\begin{tabular}{lllllll}
\hline 1.22 & 4.61 & 16.63 & 13.24 & 17.85 & 2.49 & 0.69 \\
2.61 & 4.88 & 16.31 & 14.04 & 18.92 & 1.06 & 0.32 \\
46.18 & & 132.77 & & 178.95 & 13.50 & \\
Df & 8.00 & & & & & \\
Alpha & 0.05 & & & & & \\
Critical Value & 15.51 & & & & & \\
\hline
\end{tabular}

Table 26. Chi square test for research question 2 (by country)

\begin{tabular}{|c|c|c|c|c|c|c|}
\hline \multirow{2}{*}{$\begin{array}{l}\text { Piracy } \\
\text { Observed }\end{array}$} & \multirow[b]{2}{*}{ Expected } & \multicolumn{3}{|c|}{ Variable $Y$} & & \\
\hline & & Observed & Expected & Total & & \\
\hline 0.70 & 4.04 & 6.59 & 3.49 & 7.29 & 2.53 & 1.46 \\
\hline 2.40 & 4.32 & 4.27 & 3.19 & 6.67 & 0.33 & 0.27 \\
\hline 1.30 & 3.43 & 4.19 & 2.63 & 5.49 & 0.85 & 0.58 \\
\hline 4.50 & 3.61 & 5.10 & 4.59 & 9.60 & 0.05 & 0.05 \\
\hline 2.20 & 14.74 & 4.19 & 3.06 & 6.39 & 0.39 & 0.31 \\
\hline 0.10 & 1.42 & 1.67 & 0.85 & 1.77 & 0.73 & 0.41 \\
\hline 0.10 & 0.79 & 0.64 & 0.35 & 0.74 & 0.21 & 0.13 \\
\hline 2.50 & 5.53 & 6.84 & 4.47 & 9.34 & 1.16 & 0.82 \\
\hline 2.80 & 2.66 & 2.42 & 2.50 & 5.22 & 0.00 & 0.00 \\
\hline 0.10 & 1.11 & 3.54 & 1.74 & 3.64 & 1.70 & 0.91 \\
\hline 2.40 & 5.66 & 5.15 & 3.61 & 7.55 & 0.60 & 0.46 \\
\hline 1.40 & 3.45 & 5.23 & 3.17 & 6.63 & 1.23 & 0.81 \\
\hline 0.60 & 1.77 & 5.21 & 2.78 & 5.81 & 1.95 & 1.13 \\
\hline 0.10 & 2.52 & 2.69 & 1.33 & 2.79 & 1.26 & 0.68 \\
\hline 0.10 & 1.84 & 3.32 & 1.64 & 3.42 & 1.59 & 0.85 \\
\hline 0.50 & 12.76 & 3.85 & 2.08 & 4.35 & 1.38 & 0.81 \\
\hline 1.20 & 2.71 & 6.67 & 3.76 & 7.87 & 2.06 & 1.27 \\
\hline 26.60 & 12.30 & 4.99 & 15.11 & 31.59 & 6.22 & 20.53 \\
\hline 0.10 & 1.70 & 4.50 & 2.20 & 4.60 & 2.20 & 1.18 \\
\hline 0.70 & 1.75 & 5.43 & 2.93 & 6.13 & 1.95 & 1.15 \\
\hline 48.90 & 13.74 & 0.00 & 23.39 & 48.90 & 21.45 & 0.00 \\
\hline 3.50 & 2.55 & 6.70 & 4.88 & 10.20 & 0.62 & 0.49 \\
\hline 3.40 & 1.80 & 4.19 & 3.63 & 7.59 & 0.08 & 0.07 \\
\hline
\end{tabular}




\begin{tabular}{lllll}
\hline 106.20 & & 97.38 & 203.58 & 84.94 \\
Df & 21.00 & & & \\
Alpha & 0.05 & & & \\
Critical Value & 32.67 & & & \\
\hline
\end{tabular}

Table 27. Chi square test for research question 2 (by year)

\begin{tabular}{|c|c|c|c|c|c|c|}
\hline \multirow{2}{*}{$\begin{array}{l}\text { Piracy } \\
\text { Observed }\end{array}$} & \multirow[b]{2}{*}{ Expected } & \multicolumn{3}{|c|}{ Variable $Y$} & & \\
\hline & & Observed & Expected & Total & & \\
\hline 4.65 & 3.64 & 6.15 & 5.17 & 10.80 & 0.17 & 0.16 \\
\hline 4.26 & 4.00 & 4.71 & 4.29 & 8.97 & 0.04 & 0.04 \\
\hline 5.83 & 4.64 & 2.88 & 4.17 & 8.71 & 0.36 & 0.57 \\
\hline 7.87 & 5.07 & 3.96 & 5.66 & 11.83 & 0.47 & 0.73 \\
\hline 9.43 & 5.69 & 5.18 & 6.99 & 14.61 & 0.43 & 0.63 \\
\hline 5.35 & 4.94 & 5.23 & 5.06 & 10.58 & 0.01 & 0.01 \\
\hline 3.00 & 4.28 & 5.26 & 3.95 & 8.26 & 0.40 & 0.33 \\
\hline 1.96 & 4.43 & 4.14 & 2.92 & 6.10 & 0.47 & 0.36 \\
\hline 1.22 & 4.61 & 2.09 & 1.58 & 3.31 & 0.15 & 0.12 \\
\hline 2.61 & 4.88 & 2.73 & 2.55 & 5.34 & 0.01 & 0.01 \\
\hline 46.18 & & 42.33 & & 88.51 & 5.46 & \\
\hline Df & 8.00 & & & & & \\
\hline Alpha & 0.05 & & & & & \\
\hline Critical Value & 15.51 & & & & & \\
\hline
\end{tabular}

Table 28. Chi square test for research question 3 (by country)

\begin{tabular}{lllllll}
\hline Piracy & \multicolumn{7}{c}{ Variable $\mathbf{Y}$} \\
\hline Observed & Expected & Observed & Expected & Total & & \\
\hline 0.70 & 4.04 & 0.00 & 0.42 & 0.70 & 0.62 & 0.00 \\
2.40 & 4.32 & 9.30 & 7.00 & 11.70 & 1.13 & 0.57 \\
1.30 & 3.43 & 9.03 & 6.18 & 10.33 & 1.96 & 0.90 \\
4.50 & 3.61 & 3.66 & 4.88 & 8.16 & 0.45 & 0.41 \\
2.20 & 14.74 & 5.03 & 4.32 & 7.23 & 0.17 & 0.10 \\
0.10 & 1.42 & 2.95 & 1.82 & 3.05 & 1.03 & 0.43 \\
\hline
\end{tabular}




\begin{tabular}{|c|c|c|c|c|c|c|}
\hline 0.10 & 0.79 & 0.26 & 0.22 & 0.36 & 0.01 & 0.01 \\
\hline 2.50 & 5.53 & 11.65 & 8.46 & 14.15 & 1.79 & 0.87 \\
\hline 2.80 & 2.66 & 7.58 & 6.21 & 10.38 & 0.45 & 0.25 \\
\hline 0.10 & 1.11 & 6.82 & 4.14 & 6.92 & 2.59 & 1.05 \\
\hline 2.40 & 5.66 & 5.17 & 4.53 & 7.57 & 0.14 & 0.08 \\
\hline 1.40 & 3.45 & 5.85 & 4.34 & 7.25 & 0.79 & 0.39 \\
\hline 0.60 & 1.77 & 2.40 & 1.79 & 3.00 & 0.30 & 0.15 \\
\hline 0.10 & 2.52 & 5.91 & 3.59 & 6.01 & 2.22 & 0.91 \\
\hline 0.10 & 1.84 & 6.45 & 3.92 & 6.55 & 2.44 & 1.00 \\
\hline 0.50 & 12.76 & 5.10 & 3.35 & 5.60 & 1.36 & 0.60 \\
\hline 1.20 & 2.71 & 17.59 & 11.24 & 18.79 & 5.35 & 2.30 \\
\hline 26.60 & 12.30 & 4.92 & 18.85 & 31.52 & 15.31 & 39.43 \\
\hline 0.10 & 1.70 & 0.00 & 0.06 & 0.10 & 0.09 & 0.00 \\
\hline 0.70 & 1.75 & 19.47 & 12.06 & 20.17 & 6.77 & 2.82 \\
\hline 48.90 & 13.74 & 0.00 & 29.24 & 48.90 & 43.49 & 0.00 \\
\hline 3.50 & 2.55 & 9.99 & 8.07 & 13.49 & 0.68 & 0.37 \\
\hline 3.40 & 1.80 & 18.82 & 13.29 & 22.22 & 3.43 & 1.63 \\
\hline 106.20 & & 157.95 & & 264.15 & 146.83 & \\
\hline Df & 21.00 & & & & & \\
\hline Alpha & 0.05 & & & & & \\
\hline Critical Value & 32.67 & & & & & \\
\hline
\end{tabular}

Table 29. Chi square test for research question 3 (by year)

\begin{tabular}{lllllll}
\hline Piracy & \multicolumn{7}{c}{ Variable Y } & & & \\
\hline Observed & Expected & Observed & Expected & Total & & \\
\hline 4.65 & 3.64 & 14.94 & 11.71 & 19.59 & 1.32 & 0.70 \\
4.26 & 4.00 & 7.22 & 6.86 & 11.48 & 0.03 & 0.02 \\
5.83 & 4.64 & 1.86 & 4.60 & 7.69 & 2.42 & 4.03 \\
7.87 & 5.07 & 11.24 & 11.43 & 19.11 & 0.00 & 0.00 \\
9.43 & 5.69 & 10.27 & 11.78 & 19.70 & 0.29 & 0.22 \\
5.35 & 4.94 & 10.05 & 9.21 & 15.40 & 0.11 & 0.07 \\
3.00 & 4.28 & 7.99 & 6.57 & 10.99 & 0.46 & 0.25 \\
1.96 & 4.43 & 2.68 & 2.77 & 4.64 & 0.00 & 0.00 \\
\hline
\end{tabular}




\begin{tabular}{lllllll}
\hline 1.22 & 4.61 & 1.09 & 1.38 & 2.31 & 0.09 & 0.08 \\
2.61 & 4.88 & 1.32 & 2.35 & 3.93 & 0.67 & 0.80 \\
46.18 & & 68.66 & & 114.84 & 11.58 & \\
Df & 8.00 & & & & & \\
Alpha & 0.05 & & & & & \\
Critical Value & 15.51 & & & & & \\
\hline
\end{tabular}

Table 30. Chi square test for research question 4 (by country)

\begin{tabular}{|c|c|c|c|c|c|c|}
\hline \multirow{2}{*}{$\begin{array}{l}\text { Piracy } \\
\text { Observed }\end{array}$} & \multirow[b]{2}{*}{ Expected } & \multicolumn{3}{|c|}{ Variable Y } & & \\
\hline & & Observed & Expected & Total & & \\
\hline 0.70 & 4.04 & 56.59 & 50.28 & 57.29 & 5.68 & 0.70 \\
\hline 2.40 & 4.32 & 25.46 & 24.45 & 27.86 & 0.30 & 0.04 \\
\hline 1.30 & 3.43 & 20.34 & 18.99 & 21.64 & 0.69 & 0.09 \\
\hline 4.50 & 3.61 & 55.51 & 52.66 & 60.01 & 1.10 & 0.15 \\
\hline 2.20 & 14.74 & 20.14 & 19.60 & 22.34 & 0.10 & 0.01 \\
\hline 0.10 & 1.42 & 73.44 & 64.54 & 73.54 & 8.81 & 1.08 \\
\hline 0.10 & 0.79 & 3.39 & 3.06 & 3.49 & 0.25 & 0.03 \\
\hline 2.50 & 5.53 & 34.39 & 32.37 & 36.89 & 0.90 & 0.12 \\
\hline 2.80 & 2.66 & 28.56 & 27.52 & 31.36 & 0.28 & 0.04 \\
\hline 0.10 & 1.11 & 21.53 & 18.98 & 21.63 & 2.45 & 0.30 \\
\hline 2.40 & 5.66 & 44.96 & 41.56 & 47.36 & 1.99 & 0.26 \\
\hline 1.40 & 3.45 & 19.86 & 18.66 & 21.26 & 0.56 & 0.07 \\
\hline 0.60 & 1.77 & 30.24 & 27.06 & 30.84 & 2.67 & 0.33 \\
\hline 0.10 & 2.52 & 28.80 & 25.36 & 28.90 & 3.34 & 0.41 \\
\hline 0.10 & 1.84 & 45.48 & 40.00 & 45.58 & 5.38 & 0.66 \\
\hline 0.50 & 12.76 & 33.63 & 29.95 & 34.13 & 3.24 & 0.40 \\
\hline 1.20 & 2.71 & 31.82 & 28.98 & 33.02 & 2.00 & 0.25 \\
\hline 26.60 & 12.30 & 23.95 & 44.36 & 50.55 & 67.30 & 17.39 \\
\hline 0.10 & 1.70 & 75.09 & 65.98 & 75.19 & 9.01 & 1.10 \\
\hline 0.70 & 1.75 & 21.04 & 19.08 & 21.74 & 1.45 & 0.18 \\
\hline 48.90 & 13.74 & 5.84 & 48.04 & 54.74 & 265.66 & 304.90 \\
\hline 3.50 & 2.55 & 19.20 & 19.92 & 22.70 & 0.19 & 0.03 \\
\hline 3.40 & 1.80 & 41.86 & 39.72 & 45.26 & 0.83 & 0.11 \\
\hline
\end{tabular}




\begin{tabular}{lllll}
\hline 106.20 & & 761.12 & 867.32 & 712.85 \\
Df & 21.00 & & & \\
Alpha & 0.05 & & & \\
Critical Value & 32.67 & & & \\
\hline
\end{tabular}

Table 31. Chi square test for research question 4 (by year)

\begin{tabular}{lllllll}
\hline Piracy & \multicolumn{7}{c}{ Variable Y } & & & \\
\hline Observed & Expected & Observed & Expected & Total & & \\
\hline 4.65 & 3.64 & 37.36 & 36.87 & 42.01 & 0.05 & 0.01 \\
4.26 & 4.00 & 36.44 & 35.72 & 40.70 & 0.11 & 0.01 \\
5.83 & 4.64 & 31.84 & 33.06 & 37.67 & 0.32 & 0.05 \\
7.87 & 5.07 & 34.00 & 36.74 & 41.87 & 1.47 & 0.22 \\
9.43 & 5.69 & 35.97 & 39.84 & 45.40 & 2.69 & 0.42 \\
5.35 & 4.94 & 35.45 & 35.80 & 40.80 & 0.03 & 0.00 \\
3.00 & 4.28 & 33.98 & 32.45 & 36.98 & 0.52 & 0.07 \\
1.96 & 4.43 & 32.93 & 30.62 & 34.89 & 1.25 & 0.16 \\
1.22 & 4.61 & 27.14 & 24.89 & 28.36 & 1.46 & 0.19 \\
2.61 & 4.88 & 25.81 & 24.94 & 28.42 & 0.22 & 0.03 \\
46.18 & & 330.92 & & 377.10 & 9.26 & \\
Df & 8.00 & & & & & \\
Alpha & 0.05 & & & & & \\
Critical Value & 15.51 & & & & & \\
\hline
\end{tabular}

Table 32. Chi square test for research question 5 (by country)

\begin{tabular}{lllllll}
\hline Piracy & \multicolumn{5}{c}{ Variable $\mathbf{Y}$} \\
\hline Observed & Expected & Observed & Expected & Total & & \\
\hline 0.70 & 4.04 & 33.24 & 17.70 & 33.94 & 14.87 & 7.27 \\
2.40 & 4.32 & 0.00 & 1.25 & 2.40 & 1.36 & 0.00 \\
1.30 & 3.43 & 5.06 & 3.32 & 6.36 & 1.00 & 0.60 \\
4.50 & 3.61 & 20.06 & 12.81 & 24.56 & 4.48 & 2.62 \\
2.20 & 14.74 & 6.93 & 4.76 & 9.13 & 1.08 & 0.68 \\
0.10 & 1.42 & 28.70 & 15.02 & 28.80 & 13.58 & 6.52 \\
\hline
\end{tabular}




\begin{tabular}{lllllll}
\hline 0.10 & 0.79 & 0.00 & 0.05 & 0.10 & 0.06 & 0.00 \\
2.50 & 5.53 & 2.36 & 2.53 & 4.86 & 0.01 & 0.01 \\
2.80 & 2.66 & 0.00 & 1.46 & 2.80 & 1.59 & 0.00 \\
0.10 & 1.11 & 0.00 & 0.05 & 0.10 & 0.06 & 0.00 \\
2.40 & 5.66 & 2.44 & 2.52 & 4.84 & 0.00 & 0.00 \\
1.40 & 3.45 & 0.00 & 0.73 & 1.40 & 0.80 & 0.00 \\
0.60 & 1.77 & 0.00 & 0.31 & 0.60 & 0.34 & 0.00 \\
0.10 & 2.52 & 0.00 & 0.05 & 0.10 & 0.06 & 0.00 \\
0.10 & 1.84 & 3.42 & 1.84 & 3.52 & 1.49 & 0.73 \\
0.50 & 12.76 & 0.01 & 0.27 & 0.51 & 0.27 & 6.55 \\
1.20 & 2.71 & 0.10 & 0.68 & 1.30 & 0.54 & 3.34 \\
26.60 & 12.30 & 13.37 & 20.84 & 39.97 & 2.92 & 4.17 \\
0.10 & 1.70 & 0.00 & 0.05 & 0.10 & 0.06 & 0.00 \\
0.70 & 1.75 & 0.00 & 0.36 & 0.70 & 0.40 & 0.00 \\
48.90 & 13.74 & 0.00 & 25.50 & 48.90 & 27.77 & 0.00 \\
3.50 & 2.55 & 0.00 & 1.82 & 3.50 & 1.99 & 0.00 \\
3.40 & 1.80 & 0.00 & 1.77 & 3.40 & 1.93 & 0.00 \\
106.20 & & 115.69 & & 221.89 & 109.16 & \\
Df & 21.00 & & & & & \\
Alpha & 0.05 & & & & & \\
Critical Value & 32.67 & & & & & \\
\hline & & & & & & \\
\hline
\end{tabular}

Table 33. Chi square test for research question 5 (by year)

\begin{tabular}{lllllll}
\hline Piracy & \multicolumn{7}{c}{ Variable Y } & & & \\
\hline Observed & Expected & Observed & Expected & Total & & \\
\hline 4.65 & 3.64 & 7.83 & 6.51 & 12.48 & 0.29 & 0.22 \\
4.26 & 4.00 & 8.20 & 6.50 & 12.46 & 0.49 & 0.35 \\
5.83 & 4.64 & 4.29 & 5.28 & 10.12 & 0.20 & 0.23 \\
7.87 & 5.07 & 5.41 & 6.92 & 13.28 & 0.36 & 0.42 \\
9.43 & 5.69 & 6.68 & 8.40 & 16.11 & 0.38 & 0.44 \\
5.35 & 4.94 & 6.31 & 6.08 & 11.66 & 0.01 & 0.01 \\
3.00 & 4.28 & 5.28 & 4.32 & 8.28 & 0.23 & 0.18 \\
1.96 & 4.43 & 4.39 & 3.31 & 6.35 & 0.38 & 0.27 \\
1.22 & 4.61 & 1.90 & 1.63 & 3.12 & 0.05 & 0.04 \\
\hline
\end{tabular}




\begin{tabular}{lllllll}
\hline 2.61 & 4.88 & 0.00 & 1.36 & 2.61 & 1.48 & 0.00 \\
46.18 & & 50.29 & & 96.47 & 6.04 & \\
Df & 8.00 & & & & & \\
Alpha & 0.05 & & & & & \\
Critical Value & 15.51 & & & & & \\
\hline
\end{tabular}

\section{Conclusions}

Research question 1 stated: To what extent does piracy impact the Gulf of Guinea in relation to the Liner Shipping connectivity index? The hypothesis was: Piracy has a significant impact on the Gulf of Guinea in relation to the Liner Shipping connectivity index. The null hypothesis was: Piracy has no impact on the Gulf of Guinea in relation to the Liner Shipping connectivity index. The chi square test for countries showed that the null hypothesis was rejected, suggesting that countries are greatly impacted. However, the chi square test for years showed that the null hypothesis was accepted, suggesting that in the long run, piracy attacks have no impact on the Liner Shipping connectivity index.

Research question 2 stated: To what extent does piracy impact the Gulf of Guinea in relation to gross domestic product growth rate? The hypothesis was: Piracy has a significant impact on the Gulf of Guinea in relation to gross domestic product growth rate. The null hypothesis was: Piracy has no impact on the Gulf of Guinea in relation to gross domestic product growth rate. The chi square test for countries showed that the null hypothesis was rejected, suggesting that countries are greatly impacted. However, the chi square test for years showed that the null hypothesis was accepted, suggesting that in the long run, piracy attacks have no impact on the gross domestic product growth rate.

Research question 3 stated: To what extent does piracy impact the Gulf of Guinea in relation to imports growth rate? The hypothesis was: Piracy has a significant impact on the Gulf of Guinea in relation to imports growth rate. The null hypothesis was: Piracy has no impact on the Gulf of Guinea in relation to imports growth rate. The chi square test for countries showed that the null hypothesis was rejected, suggesting that countries are greatly impacted. However, the chi square test for years showed that the null hypothesis was accepted, suggesting that in the long run, piracy attacks have no impact on the imports growth rate.

Research question 4 stated: To what extent does piracy impact the Gulf of Guinea in relation to exports as a percent of gross domestic product? The hypothesis was: Piracy has a significant impact on the Gulf of Guinea in relation to exports as a percent of gross domestic product. The null hypothesis was: Piracy has no impact on the Gulf of Guinea in relation to exports as a percent of gross domestic product. The chi square test for countries showed that the null hypothesis was rejected, suggesting that countries are greatly impacted. However, the chi square test for years showed that the null hypothesis was accepted, suggesting that in the long run, piracy attacks have no imports on exports as a percent of gross domestic product. 


\section{Macrothink}

The final research question stated: To what extent does piracy impact the Gulf of Guinea in relation to oil production as oil rents as a percent of gross domestic product? The hypothesis was: Piracy has a significant impact on the Gulf of Guinea in relation to oil production as oil rents as a percent of gross domestic product. The null hypothesis was: Piracy has no impact on the Gulf of Guinea in relation to oil production as oil rents as a percent of gross domestic product. The chi square test for countries showed that the null hypothesis was rejected, suggesting that countries are greatly impacted. However, the chi square test for years showed that the null hypothesis was accepted, suggesting that in the long run, piracy attacks have no impact on the oil productions as oil rents as a percent of gross domestic product.

Inferences: The Gulf of Guinea is a very significant region endowed with huge reserve of natural resources. These natural resources also consist of large deposits of oil and natural gas. This region is fertile ground for illegal, unreported, unregulated (IUU) fishing due to its vast pristine fishing grounds that draws in several fleets of vessels to do fishing. From the literature it is worth noting the effects on maritime transport and security. Shipping does not operate in a vacuum but will thrive in a security assured domain. By the literature, it is necessary to determine also the impacts vis à vis maritime transportation and security and how some economic indicators were impacted by pirate attacks.

As a lasting solution to curtail the incidence of piracy the study observed it prudent to end all forms of financial help to nations keeping or harbouring pirates. Governments of the Gulf of Guinea are to exhibit good governance to bring domestic satisfaction and prevent any nefarious activities like piracy form happening. More piracy monitoring stations should be employed to maintain effective listening and monitoring. Patrolling of their seas must be done by their navies and they must be well equipped. There should be more naval collaboration and exercises with the advanced navies to sharpen their surveillance and patrol skills. Information should flow freely and the regional block of the Gulf of Guinea should share information with other organisations like AFRICOM, INTERPOL, and NATO should be done.

\section{References}

Babbie, E. R. (2010). The practice of social research (12th ed.). Wadsworth Cengage.

Baetschmann, G., Staub, K. E., \& Winkelmann, R. (2015). Consistent estimation of the fixed effects ordered logit model. Journal of the Royal Statistical Society: Series A (Statistics in Society), 178(3), 685-703. https://doi.org/10.1111/rssa.12090

Belykh, A. A. (1989). A note on the origins of input - output analysis and the contribution of the early soviet economists: Chayanov, Bogdanov and Kritsman. Soviet Studies, 41(3), 426-429. https://doi.org/10.1080/09668138908411823

Blanchard, O., \& Landier, A. (2002). The Perverse Effects of Partial Labour Market Reform: Fixed-Term Contracts in France. The Economic Journal, 112(480), F214-F244. https://doi.org/10.1111/1468-0297.00047

Bowden, A. The Economic Cost of Maritime Piracy (2010). One Earth Future Foundation. 
Bradford, J. F. (2005). The growing prospects for maritime security cooperation in Southeast Asia. Naval War College Review, 58(3), 63-88.

Chalk, P. (2009). Maritime Piracy: Reasons, Dangers and Solutions. Retrieved from https://trid.trb.org/view.aspx?id=968732

Chen, Y.-Y., Schmidt, P., \& Wang, H.-J. (2014). Consistent estimation of the fixed effects stochastic frontier model. Journal of Econometrics, 181(2), 65-76. https://doi.org/10.1016/j.jeconom.2013.05.009

Chi Square Test. (n.d.). Retrieved July 15, 2015, from http://www2.lv.psu.edu/jxm57/irp/chisquar.html

Clark, D. L. (1984). Planning and the real origins of input-output analysis. Journal of Contemporary Asia, 14(4), 408-429. https://doi.org/10.1080/00472338485390301

Clark, K. K. (2009). Maritime piracy: nature, impact and legal frameworks for prosecution. International Journal of Criminal Justice Sciences, 4(1), 13-22.

Coggins, B. L. (2012). Global patterns of maritime piracy, 2000-09. Journal of Peace Research, 49(4), 605-617. https://doi.org/10.1177/0022343312442520

Collier, P. (2007). The Bottom Billion: Why the Poorest Countries Are Failing and What Can Be Done About It. Oxford: Oxford University Press.

Computing the Pearson Correlation Coefficient. (2003). Retrieved June 10, 2015, from http://www.stat.wmich.edu/s216/book/node122.html

Critical Values of the Chi-Square Distribution. (n.d.) (Vol. 2015 SRC).

Data Analysis - Pearson's Correlation Coefficient. (2015). Retrieved from http://learntech.uwe.ac.uk/da/Default.aspx?pageid=1442

Distance Calculator. (2017). Distance Calculations -- Countries to New Guinea. Retrieved from https://www.distancecalculator.net/countries

Germond, B. (2011). The EU's security and the sea: defining a maritime security strategy. European Security, 20(4), 563-584. https://doi.org/10.1080/09662839.2011.635648

Gilpin, R. (2007). Enhancing Maritime Security in the Gulf of Guinea. Monterey, CA: Naval Postgraduate School Monterey CA Center for Contemporary Conflict.

Gjelsvik, I. M., \& Bjørgo, T. (2012). Ex-pirates in Somalia: processes of engagement, disengagement, and reintegration. Journal of Scandinavian Studies in Criminology and Crime Prevention, 13(2), 94-114. https://doi.org/10.1080/14043858.2012.729353

Hansen, S. J. (2012). The dynamics of Somali piracy. Studies in Conflict and Terrorism, $35(7 / 8), 523-530$.

Hausman, J. A., \& de Leeuw, J. (1978). Specification Tests in Econometrics. Econometrica, 46(6), 1251. https://doi.org/10.2307/1913827 
Hebl, M. (2015). Descriptive Statistics. Retrieved from http://onlinestatbook.com/2/introduction/descriptive.html

International Maritime Bureau. (2008). Piracy and Armed Robbery against Ships Report: Report for the Period 1 January - 31 March 2008.

International Maritime Bureau. (2010). Piracy and Armed Robbery Against Ships: Annual Report.

International Maritime Bureau. (2015). International Maritime Bureau. Retrieved from https://icc-ccs.org/icc/imb

International Maritime Bureau. (2016). Piracy and armed robbery against ships.

Loucks, W. N. (William N., \& Whitney, W. G.,1973). Comparative economic systems (9th ed.). Harper \& Row.

Luft, G., Korin A. (2004). Terrorism on the High Seas. Foreign Affairs, 83(6), 61-71.

Mann, P. S. (1995). Introductory statistics (2nd ed.). Wiley.

McHugh, M. L. (2013). The chi-square test of independence. Biochemica Medica, 23(2), 143-149. https://doi.org/10.11613/BM.2013.018

Neethling, T. (2010). Piracy around Africa's west and east coasts: a comparative political perspective. Scientia Militaria: South African Journal of Military Studies, 38(2), 89-108.

Nick, T. G. (2007). Descriptive Statistics. In Methods in Molecular Biology (pp. 33-52). New York: Springer. https://doi.org/10.1007/978-1-59745-530-5_3

Nincic, D. (2009). Maritime piracy in Africa: The humanitarian dimension. African Security Review, 18(3), 1-16. https://doi.org/10.1080/10246029.2009.9627538

Onuoha, F. (2009). Sea piracy and maritime security in the Horn of Africa: The Somali coast and Gulf of Aden in perspective. African Security Review, 18(3), 31-44. https://doi.org/10.1080/10246029.2009.9627540

Osinowo, A. A. (2015). Combating Piracy in the Gulf of Guinea.

Penn State Science. (2015). Statistical Significance of Observed Relationship/Chi-Square Test. Retrieved May 10, 2015, from https://onlinecourses.science.psu.edu/stat200/node/73

Ramsbotham, Oliver, (2011). Contemporary Conflict Resolution. Cambridge: Polity Press

Rosenberg, D., \& Chung, C. (2008). Maritime Security in the South China Sea: Coordinating Coastal and User State Priorities. Ocean Development and International Law, 39(51). https://doi.org/10.1080/00908320701641602

Samuels, R. J. (2008). "New Fighting Power!" Japan's Growing Maritime Capabilities and East Asian Security. International Security, 32(3), 84-112. https://doi.org/10.1162/isec.2008.32.3.84 
Sedgwick, P. (2012). Pearson's correlation coefficient. BMJ, 345(7). https://doi.org/10.1136/bmj.e4483

Subramanian, S., Jones, K., Kaddour, A., \& Krieger, N. (2009). Response: The value of a historically informed multilevel analysis of Robinson's data. International Journal of Epidemiology, 38(2), 370-373. https://doi.org/10.1093/ije/dyn354

Sullivan, A. K. (2010). Piracy in the Horn of Africa and its effects on the global supply chain. Journal of Transportation Security, 3(4), 231-243. https://doi.org/10.1007/s12198-010-0049-9

Tepp, E. (2012). The Gulf of Guinea: Military and Non-Military Ways of Combatting Piracy. Baltic Security \& Defense Review, 14(1), 181-214.

Test, O. (2015). Your chi-square test is statistically significant \pm now wha. Pract Assess Res Eval, 20(8), 2-10.

Treves, T. (2009). Piracy, law of the sea, and use of force: developments off the coast of Somalia. European Journal of International Law, 20(2), 399-414. https://doi.org/10.1093/ejil/chp027

Trochim, W. M. K. (2006). Descriptive statistics. Retrieved from http://www.socialresearchmethods.net/kb/statdesc.php

UNCTAD. (2017). Liner Shipping Connectivity Index. Retrieved from http://unctadstat.unctad.org/wds/TableViewer/tableView.aspx?ReportId=92

University of Regina. (n.d.). Ch. 10 -- Chi Square Test. Retrieved September 26, 2015, from http://uregina.ca/ gingrich/ch10.pdf

Vreÿ, F. (2009). Bad order at sea: From the Gulf of Aden to the Gulf of Guinea. African Security Review, 18(3), 17-30. https://doi.org/10.1080/10246029.2009.9627539

World Bank. (2017). World Development Indicators. Retrieved from http://databank.worldbank.org/data/reports.aspx?source=2\&country=SLE\#

Yang, Z. L., Wang, J., Bonsall, S., \& Fang, Q. G. (2009). Use of Fuzzy Evidential Reasoning in Maritime Security Assessment. Risk Analysis, 29(1), 95-120. https://doi.org/10.1111/j.1539-6924.2008.01158.x

\section{Notes}

Note 1. For all research questions, one of the variables is piracy attacks. Variable Y refers to the other corresponding

Note 2. The maximum value is found in the descriptive statistics section..

Note 3. Excel function: = AVERAGE(RANGE).

Note 4. Excel function: = MEDIAN(RANGE); = MIN(RANGE); and = MAX(RANGE). 
Note 5. Excel function: = STDEV.S (RANGE).

\section{Copyright Disclaimer}

Copyright for this article is retained by the author(s), with first publication rights granted to the journal.

This is an open-access article distributed under the terms and conditions of the Creative Commons Attribution license (http://creativecommons.org/licenses/by/4.0/). 